\title{
Symmetric Intuitionistic Fuzzy Weighted Mean Operators Based on Weighted Archimedean $t$-Norms and $t$-Conorms for Multi-Criteria Decision Making
}

\author{
Zhen Ming MA*, Wei YANG \\ School of Mathematics and Statistics, Linyi University, Linyi 276005, China \\ e-mail:dmgywto@126.com,wyoeng@126.com
}

Received: February 2019; accepted: September 2019

\begin{abstract}
Using different operational laws on membership and non-membership information, various intuitionistic fuzzy aggregation operators based on Archimedean $t$-norm and $t$-conorm or their special cases have been extensively investigated for multi-criteria decision making. In spite of this, they are not suitable for some practical cases. In this paper, symmetric intuitionistic fuzzy weighted mean operators w.r.t. general weighted Archimedean $t$-norms and $t$-conorms are introduced to deal neutrally or fairly with membership and non-membership information to meet the need of decision makers in some cases. The relationship among the proposed operators and the existing ones is discussed. Particularly, using the parameters in the aggregation operators, the attitude whether the decision maker is optimistic, pessimistic or impartial is reflected. At last, an example is given to show the behaviour of the proposed operators for multi-criteria decision making under intuitionistic fuzzy environment.
\end{abstract}

Key words: multi-criteria decision making, intuitionistic fuzzy set, weighted Archimedean $t$-norm and $t$-conorm, symmetric intuitionistic fuzzy weighted mean operator.

\section{Introduction}

Since the introduction of fuzzy sets by Zadeh (1965), various generalizations of fuzzy sets have been provided, such as intuitionistic fuzzy sets introduced by Atanassov (1986), abbreviated here as A-IFS (the reasons for this are presented in Dubois et al., 2005), grey set (Deng, 1989), vague set (Gau and Buehrer, 1993), interval-valued fuzzy set (Sambuc, 1975; Zadeh, 1975), and so on. Deschrijver and Kerre (2003, 2007) investigated the relationships among some extensions of fuzzy set theory, and proved that A-IFS, grey set, vague set and interval-valued fuzzy set are equivalent. As we know, it is constructed through the membership degree, the non-membership degree and the hesitancy degree, which can describe the uncertainty and fuzziness more objectively than the usual fuzzy

\footnotetext{
* Corresponding author.
} 
set. Thus A-IFS has attracted more and more attention from researchers and has been used to solve many problems, especially the multi-criteria decision making (MCDM) problems.

An effective method to deal with the MCDM problem under intuitionistic fuzzy environment is to calculate the aggregation values of the alternatives. At present, plenty of aggregation operators in fuzzy environment have been extended to fit different situations in intuitionistic fuzzy case. All kinds of mean operators, such as quasi-arithmetic means (Hardy et al., 1934), (generalized) Bonferroni mean (BM, GBM) (Bonferroni, 1950; Yager, 2009) and (induced) ordered weighted averaging (OWA, IOWA) operators (Yager, 1988; Yager and Filev, 1999), are hot topics in aggregation, and a lot of related work has been done. With respect to the operations defined for intuitionistic fuzzy numbers (IFNs) based on algebraic product $t$-norm, probabilistic sum $t$-conorm and OWA operator, $\mathrm{Xu}$ and Yager (2006), Xu (2007) generalized the weighted geometric averaging operator to the intuitionistic fuzzy weighted geometric/averaging (IFWG/IFWA) operator, the intuitionistic fuzzy ordered weighted geometric/averaging (IFO WG/IFOWA) operator and the intuitionistic fuzzy hybrid geometric/averaging (IFHG/IFHA) operator and applied them to the MCDM problem under intuitionistic fuzzy environment. Although the IFHA (IFHG) operator generalized both the IFWA (IFWG) and IFOWA (IFOWG) operators by weighting the given importance and the ordered position of the arguments, there is a flaw pointed out by Liao and $\mathrm{Xu}$ (2014) that these hybrid aggregation operators do not satisfy some desirable properties, such as boundedness and idempotency; the developed operators not only can weigh both the arguments and their ordered positions simultaneously, but also have some desirable properties, such as idempotency, boundedness, and monotonicity. Based on the generalized OWA operator proposed by Yager (2004b), Li (2010) and Zhao et al. (2010) introduced the generalized IFWA, generalized IFOWA, and generalized IFHA operators, and applied them to multiple attribute decision making with intuitionistic fuzzy information. Using the operations defined in Xu (2007), Xu and Yager (2011) investigated the BM under intuitionistic fuzzy environments, developed an intuitionistic fuzzy BM (IFBM) and discussed its variety of special cases. Then, they applied the weighted IFBM to multicriteria decision making. Considering that the algebraic product and Einstein $t$-norms are two prototypical examples of the class of strict Archimedean $t$-norms (Klement et al., 2000), Wang and Liu $(2011,2012)$ proposed the intuitionistic fuzzy Einstein weighted geometric/averaging (IFEWG/IFEWA) operator and the intuitionistic fuzzy Einstein ordered weighted geometric/averaging (IFEOWG/IFEOWA) operator. By using Archimedean $t$-norm and $t$-conorm, Xia et al. (2012) defined the Archimedean $t$-norm and $t$-conorm based intuitionistic fuzzy weighted averaging (ATS-IFWA) operator and the Archimedean $t$-norm and $t$-conorm based intuitionistic fuzzy geometric (ATS-IFWG) operator to provide more choices for the decision makers by these parameterized $t$-norms and $t$-conorms. By extending the quasi-arithmetic ordered weighted averaging operator to different intuitionistic fuzzy situations, Yang and Chen (2012) introduced three kinds of new operators: the quasi-IFOWA operator, the quasi-intuitionistic fuzzy Choquet ordered averaging operator and the quasi-IFOWA operator based on the Dempster-Shafer belief structure. Tan et al. (2013) provided a critical analysis of Yang and Chen's operations to elicit their disadvantages, and associating with operations in Xia et al. (2012), proposed 
a new quasi-IFOWA operator based on Archimedean $t$-norm and $t$-conorm to overcome these faults, and obtained some consistent conclusions. Beliakov et al. (2011) declared that the IFWA operator is not consistent with the limiting case of ordinary fuzzy sets, which is undesirable, and proposed a new construction method for the IFWA operator based on the Lukasiewicz $t$-norm, which is consistent with operations on ordinary fuzzy sets. Similarly to IFBM, Beliakov and James (2013) presented two alternative methods to extend the generalized Bonferroni mean to intuitionistic fuzzy sets. Particularly, they constructed a general form of intuitionistic fuzzy aggregation operators by pairing the usual aggregation operators and their duals. Since the above intuitionistic fuzzy aggregation operators are using different aggregation operators on membership and non-membership information, it was pointed out in Xia and Xu (2012) that it is necessary to develop some neutral aggregation operators in order to be neutral in some cases and to be treated fairly. For example, it is pointed out in Xu and Ma (2019), Yang et al. (2019) that when aggregating some individual intuitionistic fuzzy preference relations into a collective one, such operators are necessary. Based on algebraic product $t$-norm, new intuitionistic fuzy aggregation operators, which treat the membership and non-membership information fairly, were defined (Liao and $\mathrm{Xu}, 2015$ ). Furthermore, due to the absence of parameters in these $t$-norms, the existing neutral aggregation operators can not provide more choices for the decision makers. Motivated by the idea of Beliakov and James (2013), Calvo and Mesiar (2003), Tan et al. (2013), Xia and Xu (2012), Xia et al. (2012), in this paper, some new intuitionistic fuzzy aggregation operators based on weighted Archimedean $t$-norm and $t$-conorm, which fairly treat membership and non-membership information and provide more choices for the decision maker, are developed.

To do so, the remainder of this paper is organized as follows: The basic concepts of weighted Archimedean $t$-norms and $t$-conorms and intuitionistic fuzzy sets are introduced in Section 2. In Sections 3 and 4, symmetric intuitionistic fuzzy weighted mean operators w.r.t. weighted Archimedean $t$-norms and $t$-conorms are defined, in which using parameters the attitude whether the decision maker is optimistic, pessimistic or impartial is reflected and the relationship among the proposed operators and the existing ones is discussed. Section 5 provides an example to illustrate the behaviour of the proposed operators. In the final section, our research is concluded.

\section{Preliminaries}

To make the presentation self-contained, in what follows, we review some basic concepts.

\subsection{Weighted Archimedean $t$-Norms and $t$-Conorms}

Definition 2.1 (See Klement et al., 2000). A triangular norm ( $t$-norm) is a binary operation $T$ on the unit interval $[0,1], T:[0,1]^{2} \longrightarrow[0,1]$, such that for all $x, y, z \in[0,1]$ :

(T1) $T(x, T(y, z))=T(T(x, y), z)$,

(T2) $T(x, y)=T(y, x)$, 
(T3) if $x \leqslant y$, then $T(x, z) \leqslant T(y, z)$,

(T4) $T(x, 1)=x$.

Definition 2.2 (See Klement et al., 2000). A triangular conorm ( $t$-conorm) is a binary operation $S$ on the unit interval $[0,1], S:[0,1]^{2} \longrightarrow[0,1]$, which, for all $x, y, z \in[0,1]$, satisfies (T1)-(T3) and

(S4) $S(x, 0)=x$ for all $x \in[0,1]$.

Definition 2.3 (See Klement et al., 2000). A $t$-norm $T$ is called an Archimedean $t$-norm if it is continuous and $T(x, x)<x$ for all $x \in(0,1)$. An Archimedean $t$-norm is called a strictly Archimedean $t$-norm if it is strictly increasing in each variable for $x, y \in(0,1)$.

Definition 2.4 (See Klement et al., 2000). A $t$-conorm $S$ is called an Archimedean $t$ conorm if it is continuous and $S(x, x)>x$ for all $x \in(0,1)$. An Archimedean $t$-conorm is called a strictly Archimedean $t$-conorm if it is strictly increasing in each variable for $x, y \in(0,1)$.

It is well known (see Klement et al., 2000) that a strict Archimedean $t$-norm can be expressed via its additive generator $g$ as follows: $T(x, y)=g^{-1}(g(x)+g(y))$, and the same applies to its dual $t$-conorm, $S(x, y)=h^{-1}(h(x)+h(y))$, with $h(x)=g(1-x)$. That is, $S(x, y)=1-g^{-1}(g(1-x)+g(1-y))$. If not otherwise specified, we remind that an additive generator of a continuous Archimedean $t$-norm is a strictly decreasing function $g:[0,1] \rightarrow[0, \infty]$ such that $g(1)=0$ in the following parts. For nilpotent operations the inverse changes to the pseudo-inverse.

For a given weight vector $\omega=\left(\omega_{1}, \omega_{2}, \ldots, \omega_{n}\right)^{\top}$ of $x=\left(x_{1}, x_{2}, \ldots, x_{n}\right)$ where $\omega_{j} \in$ $[0, \infty)$ is the weight of $x_{j}(j=1,2, \ldots, n)$, we denote the weighted $t$-norm aggregation operator as $T_{\omega}(x)$. Let $T$ be a continuous Archimedean $t$-norm with an additive generator $g$ (Yager, 2004a), and we define the weighted aggregation as:

$$
T_{\omega, g}(x)=g^{-1}\left(\sum_{j=1}^{n} \omega_{j} g\left(x_{j}\right)\right), \quad S_{\omega, g}(x)=1-g^{-1}\left(\sum_{j=1}^{n} \omega_{j} g\left(1-x_{j}\right)\right) .
$$

If we assign specific forms to the function $g$, then some weighted Archimedean $t$-norm from the well-known Archimedean $t$-norms (Klement et al., 2000) can be obtained:

Let $g_{\gamma} S S(t)=\frac{1-t^{\gamma}}{\gamma}, \gamma \neq 0$, then the weighted Schweizer-Sklar $t$-norm is provided as follows:

$$
T_{\omega, g_{\gamma} S}(x)=\left(\sum_{j=1}^{n} \omega_{j} x_{j}^{\gamma}\right)^{\frac{1}{\gamma}}
$$

Particularly, if $\gamma=1$ and $\omega=(1,1)$, then Schweizer-Sklar $t$-norm reduces to the Łukasiewicz $t$-norm. 
Furthermore, let $A=H(T, S)$ be a composed aggregation operator based on a continuous $t$-norm $T$, a continuous $t$-conorm $S$ and a binary aggregation operator $H$, Calvo and Mesiar (2003) introduced weighted $t$-norms based aggregation operator $A_{\omega}$ : $[0,1]^{\operatorname{dim} \omega} \rightarrow[0,1]$ as $A_{\omega}=H\left(T_{\omega}, S_{\omega}\right)$, i.e. $A_{\omega}(x)=H\left(T_{\omega}(x), S_{\omega}(x)\right)$.

\subsection{Intuitionistic Fuzzy Sets}

Definition 2.5 (See Atanassov, 1986). Let $X$ be a given universe. An intuitionistic fuzzy set (IFS) $A$ in $X$ is defined as follows:

$$
A=\left\{x, \mu_{A}(x), v_{A}(x) \mid x \in X\right\},
$$

$\mu_{A}(x), v_{A}(x) \in[0,1]$ indicate the amounts of guaranteed membership and nonmembership of $x$ in $A$, respectively, and satisfy $\mu_{A}(x)+v_{A}(x) \leqslant 1$.

We recall for an intuitionistic fuzzy set the membership grade of $x$ in $A$ which is represented as a pair $\left(\mu_{A}(x), v_{A}(x)\right)$ is called a intuitionistic fuzzy number (IFN) $(\mathrm{Xu}, 2007)$ and the set of all IFNs is denoted as $\mathcal{I} \mathcal{F} \mathcal{N}$. Here, the expression $\pi_{A}(x)=$ $1-\mu_{A}(x)-v_{A}(x)$ is called the hesitancy of $x$. The IFN $\alpha=\left(\mu_{\alpha}, v_{\alpha}\right)$ has a physical interpretation, for example, if $\alpha=(0.3,0.2)$, then it can be interpreted as "the vote for resolution is 3 in favour, 2 against, and 5 abstentions (Gau and Buehrer, 1993). The following partial order $\leqslant$ on $\mathcal{I} \mathcal{F} \mathcal{N}$, which is defined for $\alpha=\left(\mu_{\alpha}, v_{\alpha}\right)$ and $\beta=\left(\mu_{\beta}, v_{\beta}\right)$ as $\beta \leqslant \alpha$ if and only if $\mu_{\beta} \leqslant \mu_{\alpha}$ and $v_{\alpha} \leqslant v_{\beta}$. For an IFN $\alpha$, a score function $s$ (Chen and Tan, 1994), which is defined as the difference of membership and non-membership function, can be denoted as: $s(\alpha)=\mu_{\alpha}-v_{\alpha}$, where $s(\alpha) \in[-1,1]$. The larger the score $s(\alpha)$ is, the greater the IFN $\alpha$ is. To make the comparison method more discriminatory, an accuracy function $h$ (Hong and Choi, 2000) is defined as follows: $h(\alpha)=\mu_{\alpha}+v_{\alpha}$, where $h(\alpha) \in[0,1]$. When the scores are the same, the larger the accuracy $h(\alpha)$ is, the greater the IFN $\alpha$ is. However, it is obvious that $h(\alpha)+\pi_{\alpha}=1$.

Definition 2.6 (See Xu, 2007). Let $\alpha, \beta$ be two IFNs. Then, we have

(1) If $s(\beta)>s(\alpha)$, then $\beta$ is bigger than $\alpha$, i.e. $\beta \succ \alpha$.

(2) If $s(\alpha)=s(\beta)$ :

(a) if $h(\beta)>h(\alpha)$, then $\beta$ is bigger than $\alpha$, i.e. $\beta \succ \alpha$;

(b) if $h(\alpha)=h(\beta)$, i.e. $\alpha=\beta$.

Definition 2.7 (See Beliakov et al., 2011; Xia et al., 2012). Let $\alpha_{i}=\left(\mu_{\alpha_{i}}, v_{\alpha_{i}}\right)(i=1,2)$ and $\alpha=\left(\mu_{\alpha}, v_{\alpha}\right)$ be three IFNs, then we have

(1) $\alpha_{1} \oplus \alpha_{2}=\left(1-g^{-1}\left(g\left(1-\mu_{\alpha_{1}}\right)+g\left(1-\mu_{\alpha_{2}}\right)\right), g^{-1}\left(g\left(v_{\alpha_{1}}\right)+g\left(v_{\alpha_{2}}\right)\right)\right)$;

(2) $\alpha_{1} \otimes \alpha_{2}=\left(g^{-1}\left(g\left(\mu_{\alpha_{1}}\right)+g\left(\mu_{\alpha_{2}}\right)\right), 1-g^{-1}\left(g\left(1-v_{\alpha_{1}}\right)+g\left(1-v_{\alpha_{2}}\right)\right)\right)$;

(3) $\lambda \alpha=\left(1-g^{-1}\left(\lambda g\left(1-\mu_{\alpha}\right)\right), g^{-1}\left(\lambda g\left(v_{\alpha}\right)\right)\right), \lambda>0$;

(4) $\alpha^{\lambda}=\left(g^{-1}\left(\lambda g\left(\mu_{\alpha}\right)\right), 1-g^{-1}\left(\lambda g\left(1-v_{\alpha}\right)\right)\right), \lambda>0$;

(3) $\alpha^{c}=\left(v_{\alpha}, \mu_{\alpha}\right)$. 
For convenience, if not otherwise specified, we always denote

$$
\begin{aligned}
\alpha & =\left(\alpha_{1}, \alpha_{2}, \ldots, \alpha_{n}\right), \\
\mu_{\alpha} & =\left(\mu_{\alpha_{1}}, \mu_{\alpha_{2}}, \ldots, \mu_{\alpha_{n}}\right), \quad v_{\alpha}=\left(v_{\alpha_{1}}, v_{\alpha_{2}}, \ldots, v_{\alpha_{n}}\right), \\
1-\mu_{\alpha} & =\left(1-\mu_{\alpha_{1}}, 1-\mu_{\alpha_{2}}, \ldots, 1-\mu_{\alpha_{n}}\right), \\
1-v_{\alpha} & =\left(1-v_{\alpha_{1}}, 1-v_{\alpha_{2}}, \ldots, 1-v_{\alpha_{n}}\right),
\end{aligned}
$$

for IFNs $\alpha_{j}(j=1,2, \ldots, n)$.

Definition 2.8 (See Xia et al., 2012). Let $\alpha_{j}(j=1,2, \ldots, n)$ be IFNs and $T_{\omega, g}$ be a weighted Archimedean $t$-norm with an additive generator $g$ and IFWA ${ }^{T_{\omega, g}}: \mathcal{I F}^{n} \rightarrow$ $\mathcal{I F N}$, if

$$
\operatorname{IFWA}^{T_{\omega, g}}(\alpha)=\bigoplus_{j=1}^{n} \omega_{j} \alpha_{j}=\left(1-T_{\omega, g}\left(1-\mu_{\alpha}\right), T_{\omega, g}\left(v_{\alpha}\right)\right),
$$

then IFWA ${ }^{T_{\omega, g}}$ is called an intuitionistic fuzzy weighted averaging (IFWA ${ }^{T_{\omega, g}}$ ) operator of dimension $n$ w.r.t. $T_{\omega, g}$.

Definition 2.9 (See Xia et al., 2012). Let $\alpha_{j}(j=1,2, \ldots, n)$ be IFNs and $T_{\omega, g}$ be a weighted Archimedean $t$-norm with an additive generator $g$ and $\operatorname{IFWM}^{T_{\omega, g}}: \mathcal{I F \mathcal { N }}^{n} \rightarrow$ $\mathcal{I F N}$, if

$$
\operatorname{IFWM}^{T_{\omega, g}}(\alpha)=\bigotimes_{j=1}^{n} \alpha_{j}^{\omega_{j}}=\left(T_{\omega, g}\left(\mu_{\alpha}\right), 1-T_{\omega, g}\left(1-v_{\alpha}\right)\right)
$$

then IFWM ${ }^{T_{\omega, g}}$ is called an intuitionistic fuzzy weighted mean (IFWM ${ }^{T_{\omega, g}}$ ) operator of dimension $n$ w.r.t. $T_{\omega, g}$.

However, if we assign $g(t)$ to $g_{\gamma}^{H}(t)=\ln \left(\frac{\gamma+(1-\gamma) t}{t}\right), g_{\gamma}^{S S}(t)=\frac{1-t^{\gamma}}{\gamma}$ and $g_{\gamma}^{D}(t)=$ $\left(\frac{1-t}{t}\right)^{\gamma}$, respectively, then the following families of $\operatorname{IFWM}^{T_{\omega, g}}$ operators are obtained:

$$
\begin{aligned}
& \operatorname{IFWM}^{T, g_{\gamma}^{H}}(\alpha)=\left(T_{\omega, g_{\gamma}^{H}}\left(\mu_{\alpha_{j}}\right), 1-T_{\omega, g_{\gamma}^{H}}\left(1-v_{\alpha_{j}}\right)\right), \\
& \operatorname{IFWM}^{T, g_{\gamma} S}(\alpha)=\left(T_{\omega, g_{\gamma} S}\left(\mu_{\alpha_{j}}\right), 1-T_{\omega, g_{\gamma} S}\left(1-v_{\alpha_{j}}\right)\right), \\
& \operatorname{IFWM}^{T \omega, g_{\gamma}^{D}}(\alpha)=\left(T_{\omega, g_{\gamma} D}\left(\mu_{\alpha_{j}}\right), 1-T_{\omega, g_{\gamma} D}\left(1-v_{\alpha_{j}}\right)\right) .
\end{aligned}
$$

Note that IFWM ${ }^{T \omega, g_{\gamma}^{H}}$ has been investigated in Xia et al. (2012). In particular, if $\gamma=1$, then 


$$
\begin{aligned}
\operatorname{IFWM}^{T}{ }^{T, g_{1}^{H}}(\alpha) & =\left(\prod_{j=1}^{n} \mu_{\alpha_{j}}^{\omega_{j}}, 1-\prod_{j=1}^{n}\left(1-v_{\alpha_{j}}\right)^{\omega_{j}}\right), \\
\operatorname{IFWM}^{T}{ }^{T, g_{1}^{S S}}(\alpha) & =\left(\sum_{j=1}^{n} \omega_{j} \mu_{\alpha_{j}}, \sum_{j=1}^{n} \omega_{j} v_{\alpha_{j}}\right), \\
\operatorname{IFWM}^{T}{ }^{T, g_{1} D}(\alpha) & =\left(\left(\sum_{j=1}^{n} \omega_{j} \mu_{\alpha_{j}}^{-1}\right)^{-1}, 1-\left(\sum_{j=1}^{n} \omega_{j}\left(1-v_{\alpha_{j}}\right)^{-1}\right)^{-1}\right),
\end{aligned}
$$

$\mathrm{IFWM}^{T, g_{1}^{H}}=\mathrm{IFWG}_{\omega}$ defined by Xu (2007), IFWM ${ }^{T, g_{1}^{S S}}=\mathrm{IFWM}_{\omega}$ defined by Beliakov et al. (2011) and IFWM ${ }^{T}{ }_{\omega, g_{1}}^{D}$ are natural generalizations of fuzzy weighted geometric mean, arithmetic mean and Harmonic mean, respectively.

However, Deschrijver and Kerre (2008) provided a natural extension of an aggregation function to the environment of interval-valued fuzzy set, and Beliakov and James (2013) gave the definition for A-IFS representation as follows:

Definition 2.10 (See Beliakov and James, 2013). Given an aggregation function agg : $[0,1]^{n} \rightarrow[0,1]$, the natural extension of the aggregation function agg is given by Agg : $\mathcal{I F}^{n} \mathcal{N}^{n} \mathcal{I F \mathcal { N }}, \operatorname{Agg}(\alpha)=\left(\operatorname{agg}\left(\mu_{\alpha}\right), 1-\operatorname{agg}\left(1-v_{\alpha}\right)\right)$

\section{Symmetric Intuitionistic Fuzzy Weighted Mean Operators w.r.t. Weighted Archimedean $t$-Norms and $t$-Conorms}

Considering the work of Beliakov and James (2013), Calvo and Mesiar (2003), Tan et al. (2013), Xia and Xu (2012), Xia et al. (2012), in this section we develop some symmetric intuitionistic fuzzy weighted mean operators w.r.t. weighted Archimedean $t$-norms and $t$-conorms to fairly treat membership and non-membership information and provide more choices for the decision maker by considering his/her attitude with parameters.

\subsection{Symmetric Intuitionistic Fuzzy Weighted Mean Operators w.r.t. Weighted Archimedean $t$-Norms}

Lemma 3.1. Let $\alpha_{j}(j=1,2, \ldots, n)$ be a collection of IFNs and $T_{\omega, g}$ be a weighted Archimedean $t$-norm with an additive generator $g$. Then

$$
\left(\frac{T_{\omega, g}\left(\mu_{\alpha}\right)}{T_{\omega, g}\left(\mu_{\alpha}\right)+T_{\omega, g}\left(1-\mu_{\alpha}\right)}, \frac{T_{\omega, g}\left(v_{\alpha}\right)}{T_{\omega, g}\left(v_{\alpha}\right)+T_{\omega, g}\left(1-v_{\alpha}\right)}\right)
$$

is an IFN.

Proof. Since $\alpha_{j}(j=1,2, \ldots, n)$ are IFNs, it holds that $\mu_{\alpha_{j}}+v_{\alpha_{j}} \leqslant 1$. The antitonicity of $g$ leads that $T_{\omega, g}\left(v_{\alpha}\right) \leqslant T_{\omega, g}\left(1-\mu_{\alpha}\right)$. Thus we have $\frac{T_{\omega, g}\left(\mu_{\alpha}\right)}{T_{\omega, g}\left(\mu_{\alpha}\right)+T_{\omega, g}\left(1-\mu_{\alpha}\right)} \leqslant$ 
$\frac{T_{\omega, g}\left(\mu_{\alpha}\right)}{T_{\omega, g}\left(\mu_{\alpha}\right)+T_{\omega, g}\left(v_{\alpha}\right)}$. Similarly, it holds that $\frac{T_{\omega, g}\left(v_{\alpha}\right)}{T_{\omega, g}\left(v_{\alpha}\right)+T_{\omega, g}\left(1-v_{\alpha}\right)} \leqslant \frac{T_{\omega, g}\left(v_{\alpha}\right)}{T_{\omega, g}\left(v_{\alpha}\right)+T_{\omega, g}\left(\mu_{\alpha}\right)}$. Then it yields immediately that $\frac{T_{\omega, g}\left(\mu_{\alpha}\right)}{T_{\omega, g}\left(\mu_{\alpha}\right)+T_{\omega, g}\left(1-\mu_{\alpha}\right)}+\frac{T_{\omega, g}\left(v_{\alpha}\right)}{T_{\omega, g}\left(v_{\alpha}\right)+T_{\omega, g}\left(1-v_{\alpha}\right)} \leqslant 1$, i.e. Eq. (4) is an IFN.

Definition 3.2. Let $\alpha_{j}(j=1,2, \ldots, n)$ be a collection of IFNs, $T_{\omega, g}$ be a weighted Archimedean $t$-norm and $\operatorname{SIFWM}^{T_{\omega, g}}: \mathcal{I F N}^{n} \rightarrow \mathcal{I} \mathcal{F N}$, if

$$
\operatorname{SIFWM}^{T_{\omega, g}}(\alpha)=\left(\frac{T_{\omega, g}\left(\mu_{\alpha}\right)}{T_{\omega, g}\left(\mu_{\alpha}\right)+T_{\omega, g}\left(1-\mu_{\alpha}\right)}, \frac{T_{\omega, g}\left(v_{\alpha}\right)}{T_{\omega, g}\left(v_{\alpha}\right)+T_{\omega, g}\left(1-v_{\alpha}\right)}\right),
$$

then SIFWM ${ }^{T_{\omega, g}}$ is called a symmetric intuitionistic fuzzy weighted mean (SIFWM ${ }^{T_{\omega, g}}$ ) operator of dimension $n$ w.r.t. $T_{\omega, g}$.

Especially, $v_{\alpha_{j}}=1-\mu_{\alpha_{j}}$ for all $j=1,2, \ldots, n$, that is, all $\alpha_{j}$ are reduced to $\mu_{\alpha_{j}}$, respectively, then Eq. (5) has the following form:

$$
\operatorname{SIFWM}^{T_{\omega, g}}(\alpha)=\left(\frac{T_{\omega, g}\left(\mu_{\alpha}\right)}{T_{\omega, g}\left(\mu_{\alpha}\right)+T_{\omega, g}\left(1-\mu_{\alpha}\right)}, 1-\frac{T_{\omega, g}\left(\mu_{\alpha}\right)}{T_{\omega, g}\left(\mu_{\alpha}\right)+T_{\omega, g}\left(1-\mu_{\alpha}\right)}\right),
$$

which becomes both a symmetric sum operator of dimension $n$ (Beliakov et al., 2007) and a weighted $t$-norm-based aggregation operator with $H(x, y)=\frac{x}{1+x-y}$ (Calvo and Mesiar, 2003) to aggregate fuzzy information.

Proposition 3.3. Let $\alpha_{j}, \beta_{j}(j=1,2, \ldots, n)$ be two collections of IFNs with $\alpha=$ $\left(\alpha_{1}, \alpha_{2}, \ldots, \alpha_{n}\right), \beta=\left(\beta_{1}, \beta_{2}, \ldots, \beta_{n}\right)$, respectively, and $T_{\omega, g}$ be a weighted Archimedean $t$-norm with an additive generator $g$.

(1) If all $\alpha_{j}$ are equal, i.e. $\alpha_{j}=\delta=\left(\mu_{\delta}, v_{\delta}\right)$, for all $j$, then $\operatorname{SIFWM}^{T_{\omega, g}}(\alpha)=\delta$;

(2) If $\alpha_{j} \leqslant \beta_{j}$ for all $j$, then $\operatorname{SIFWM}^{T_{\omega, g}}(\alpha) \leqslant \operatorname{SIFWM}^{T_{\omega, g}}(\beta)$;

(3) Let $\alpha^{-}=\left(\min \left(\mu_{\alpha}\right), \max \left(v_{\alpha}\right)\right)$ and $\alpha^{+}=\left(\max \left(\mu_{\alpha}\right), \min \left(v_{\alpha_{j}}\right)\right)$, then $\alpha^{-} \leqslant$ $\operatorname{SIFWM}^{T_{\omega, g}}(\alpha) \leqslant \alpha^{+}$.

Proof. (1) By Definition 3.2, it holds that

$$
\begin{aligned}
\operatorname{SIFWM}^{T_{\omega, g}}(\alpha) & =\left(\frac{T_{\omega, g}\left(\mu_{\delta}\right)}{T_{\omega, g}\left(\mu_{\delta}\right)+T_{\omega, g}\left(1-\mu_{\delta}\right)}, 1-\frac{T_{\omega, g}\left(\mu_{\delta}\right)}{T_{\omega, g}\left(\mu_{\delta}\right)+T_{\omega, g}\left(1-\mu_{\delta}\right)}\right) \\
& =\left(\mu_{\delta}, v_{\delta}\right)=\delta
\end{aligned}
$$

and hence $\operatorname{SIFWM}^{T_{\omega, g}}(\alpha)=\delta$.

(2) Since $\alpha_{j} \leqslant \beta_{j}$ for all $j$, i.e. $\mu_{\alpha_{j}} \leqslant \mu_{\beta_{j}}$ and $v_{\beta_{j}} \leqslant v_{\alpha_{j}}$, we have $T_{\omega, g}\left(\mu_{\alpha}\right) \leqslant$ $T_{\omega, g}\left(\mu_{\beta}\right), T_{\omega, g}\left(1-\mu_{\beta}\right) \leqslant T_{\omega, g}\left(1-\mu_{\alpha}\right), T_{\omega, g}\left(1-\mu_{\alpha}\right) \leqslant T_{\omega, g}\left(1-\mu_{\beta}\right)$ and $T_{\omega, g}\left(\mu_{\beta}\right) \leqslant$ $T_{\omega, g}\left(\mu_{\alpha}\right)$, and hence 


$$
\begin{aligned}
\frac{T_{\omega, g}\left(\mu_{\alpha}\right)}{T_{\omega, g}\left(\mu_{\alpha}\right)+T_{\omega, g}\left(1-\mu_{\alpha}\right)} & =\frac{1}{1+\frac{T_{\omega, g}\left(1-\mu_{\alpha}\right)}{T_{\omega, g}\left(\mu_{\alpha}\right)}} \\
& \leqslant \frac{1}{1+\frac{T_{\omega, g}\left(1-\mu_{\beta}\right)}{T_{\omega, g}\left(\mu_{\beta}\right)}}=\frac{T_{\omega, g}\left(\mu_{\beta}\right)}{T_{\omega, g}\left(\mu_{\beta}\right)+T_{\omega, g}\left(1-\mu_{\beta}\right)}
\end{aligned}
$$

Similarly, it holds that $\frac{T_{\omega, g}\left(v_{\beta}\right)}{T_{\omega, g}\left(v_{\beta}\right)+T_{\omega, g}\left(1-v_{\beta}\right)} \leqslant \frac{T_{\omega, g}\left(v_{\alpha}\right)}{T_{\omega, g}\left(v_{\alpha}\right)+T_{\omega, g}\left(1-v_{\alpha}\right)}$. Thus we obtain $\operatorname{SIFWM}^{T_{\omega, g}}(\alpha) \leqslant \operatorname{SIFWM}^{T_{\omega, g}}(\beta)$.

(3) Since $\min _{j}\left\{\mu_{\alpha_{j}}\right\} \leqslant \mu_{\alpha_{j}} \leqslant \max _{j}\left\{\mu_{\alpha_{j}}\right\}$ and $\min _{j}\left\{v_{\alpha_{j}}\right\} \leqslant v_{\alpha_{j}} \leqslant \max _{j}\left\{v_{\alpha_{j}}\right\}$, it follows from (1) that

$$
\begin{aligned}
\min _{j}\left\{\mu_{\alpha_{j}}\right\} & \leqslant\left(\frac{T_{\omega, g}\left(\mu_{\alpha}\right)}{T_{\omega, g}\left(\mu_{\alpha}\right)+T_{\omega, g}\left(1-\mu_{\alpha}\right)}, 1-\frac{T_{\omega, g}\left(\mu_{\alpha}\right)}{T_{\omega, g}\left(\mu_{\alpha}\right)+T_{\omega, g}\left(1-\mu_{\alpha}\right)}\right) \\
& \leqslant \max _{j}\left\{\mu_{\alpha_{j}}\right\} \\
\min _{j}\left\{v_{\alpha_{j}}\right\} & \leqslant\left(\frac{T_{\omega, g}\left(v_{\alpha}\right)}{T_{\omega, g}\left(v_{\alpha}\right)+T_{\omega, g}\left(1-v_{\alpha}\right)}, 1-\frac{T_{\omega, g}\left(v_{\alpha}\right)}{T_{\omega, g}\left(v_{\alpha}\right)+T_{\omega, g}\left(1-v_{\alpha}\right)}\right) \\
& \leqslant \max _{j}\left\{v_{\alpha_{j}}\right\}
\end{aligned}
$$

thus we have $\alpha^{-} \leqslant \operatorname{SIFWM}^{T_{\omega, g}}(\alpha) \leqslant \alpha^{+}$.

When the additive generator $g$ is assigned to different forms, some specific intuitionistic fuzzy aggregation operators can be provided as follows:

(1) If $g(t)=g_{\gamma}^{H}(t)$, then the SIFWM ${ }^{T_{\omega, g}}$ operator is reduced to the following form:

$$
\operatorname{SIFWM}^{T_{\omega, g_{\gamma}^{H}}}(\alpha)=\left(\frac{T_{\omega, g_{\gamma}^{H}}\left(\mu_{\alpha}\right)}{T_{\omega, g_{\gamma}^{H}}\left(\mu_{\alpha}\right)+T_{\omega, g_{\gamma}^{H}}\left(1-\mu_{\alpha}\right)}, \frac{T_{\omega, g_{\gamma}^{H}}\left(v_{\alpha}\right)}{T_{\omega, g_{\gamma}^{H}}\left(v_{\alpha}\right)+T_{\omega, g_{\gamma}^{H}}\left(1-v_{\alpha}\right)}\right) .
$$

Especially, if $\gamma=1$, i.e. $g^{H_{1}}(t)=-\ln (t)$, then the $\operatorname{SIFWM}^{T, g_{1}^{H}}$ operator with $T_{\omega, g_{1}^{H}}(x)=\prod_{j=1}^{n} x_{j}^{\omega_{j}}$ is called a symmetric intuitionistic fuzzy weighted geometric (SIFWG) operator defined by Xia and Xu (2012); if $\gamma=2$, i.e. $g^{H_{2}}(t)=\ln \left(\frac{2-t}{t}\right)$, then the SIFWM ${ }^{T}{ }^{T, g_{2}^{H}}$ operator with $T_{\omega, g_{2}^{H}}(x)=\frac{2}{\prod_{j=1}^{n}\left(\frac{2}{x_{j}}-1\right)^{\omega_{j}}+1}$ is the symmetric form of IFWG operator based on the Einstein $t$-norm defined by Wang and Liu (2012); if $\gamma \rightarrow \infty$, then it holds that

$$
\begin{aligned}
\lim _{\gamma \rightarrow \infty} T_{\omega, g_{\gamma}^{H}}(x) & =\lim _{\gamma \rightarrow \infty} \frac{\gamma}{\prod_{j=1}^{n}\left(\frac{\gamma}{x_{j}}+1-\gamma\right)^{\omega_{j}}+\gamma-1} \\
& =\lim _{\gamma \rightarrow \infty}\left(\frac{\prod_{j=1}^{n}\left(\frac{\gamma}{x_{j}}+1-\gamma\right)^{\omega_{j}}}{\prod_{j=1}^{n} \gamma^{\omega_{j}}}+\frac{\gamma-1}{\gamma}\right)^{-1}
\end{aligned}
$$




$$
\begin{aligned}
& =\lim _{\gamma \rightarrow \infty}\left(\prod_{j=1}^{n}\left(\frac{1}{x_{j}}+\frac{1-\gamma}{\gamma}\right)^{\omega_{j}}+\frac{\gamma-1}{\gamma}\right)^{-1} \\
& =\left(\prod_{j=1}^{n}\left(\frac{1}{x_{j}}-1\right)^{\omega_{j}}+1\right)^{-1},
\end{aligned}
$$

thus we have

$$
\begin{aligned}
& \operatorname{SIFWM}_{\omega, g}^{T_{\infty}^{H}}(\alpha) \\
& \quad=\left(\frac{\prod_{j=1}^{n} \mu_{\alpha_{j}}^{\omega_{j}}}{\prod_{j=1}^{n}\left(1-\mu_{\alpha_{j}}\right)^{\omega_{j}}+\prod_{j=1}^{n} \mu_{\alpha_{j}}^{\omega_{j}}}, \frac{\prod_{j=1}^{n} v_{\alpha_{j}}^{\omega_{j}}}{\prod_{j=1}^{n}\left(1-v_{\alpha_{j}}\right)^{\omega_{j}}+\prod_{j=1}^{n} v_{\alpha_{j}}^{\omega_{j}}}\right),
\end{aligned}
$$

if $\gamma \rightarrow 0$, in a similar way, we have

$$
\operatorname{SIFWM}^{T} \omega_{, g_{0}^{H}}^{H}(\alpha)=\left(\frac{1}{\sum_{j=1}^{n} \frac{\omega_{j}}{\mu_{\alpha_{j}}}}, \frac{1}{\sum_{j=1}^{n} \frac{\omega_{j}}{\nu_{\alpha_{j}}}}\right)
$$

(2) If $g(t)=g_{\gamma}^{S S}(t)$, then the SIFWM ${ }^{T_{\omega, g}}$ operator is reduced to the following form:

$$
\begin{aligned}
& \operatorname{SIFWM}^{T^{\omega, g_{\gamma} S S}(\alpha)} \\
& \quad=\left(\frac{T_{\omega, g_{\gamma} S}\left(\mu_{\alpha}\right)}{T_{\omega, g_{\gamma} S S}\left(\mu_{\alpha}\right)+T_{\omega, g_{\gamma} S}\left(1-\mu_{\alpha}\right)}, \frac{T_{\omega, g_{\gamma} S}\left(v_{\alpha}\right)}{T_{\omega, g_{\gamma} S}\left(v_{\alpha}\right)+T_{\omega, g_{\gamma} S}\left(1-v_{\alpha}\right)}\right) .
\end{aligned}
$$

Particularly, if $\gamma \rightarrow \infty$, we assume that $x_{k}=\max \left\{x_{1}, x_{2}, \ldots, x_{n}\right\}, k \in\{1,2, \ldots, n\}$, then it follows from L'Hôpital's rule that

$$
\begin{aligned}
& \lim _{\gamma \rightarrow \infty} T_{\omega, g_{\gamma} S S}(x)=\lim _{\gamma \rightarrow \infty}\left(\sum_{j=1}^{n} \omega_{j} x_{j}^{\gamma}\right)^{\frac{1}{\gamma}}=\lim _{\gamma \rightarrow \infty} e^{\frac{\ln \left(\sum_{j=1}^{n} \omega_{j} x_{j}^{\gamma}\right)}{\gamma}} \\
& =\lim _{\gamma \rightarrow \infty} e^{\frac{\sum_{j=1}^{n} \omega_{j} x_{j}^{\gamma} \ln x_{j}}{\sum_{j=1}^{n} \omega_{j} x_{j}^{\gamma}}}=e^{\lim _{\gamma \rightarrow \infty} \frac{\sum_{j=1}^{n} \omega_{j} x_{j}^{\gamma} \ln x_{j}}{\sum_{j=1}^{n} \omega_{j} x_{j}^{\gamma}}}=e^{\lim _{\gamma \rightarrow \infty} \frac{\sum_{j=1}^{n} \omega_{j}\left(\frac{x_{j}}{x_{k}}\right)^{\gamma} \ln x_{j}}{\sum_{j=1}^{n} \omega_{j}\left(\frac{x_{j}}{x_{k}}\right) \gamma}} \\
& =e^{\frac{\omega_{k} \ln x_{k}}{\omega_{k}}}=x_{k},
\end{aligned}
$$

thus we get

$$
\operatorname{SIFWM}^{T}{ }_{\omega, g}^{S S}(\alpha)=\left(\frac{\max \left(\mu_{\alpha}\right)}{\max \left(\mu_{\alpha}\right)+\max \left(1-\mu_{\alpha}\right)}, \frac{\max \left(v_{\alpha}\right)}{\max \left(v_{\alpha}\right)+\max \left(1-v_{\alpha}\right)}\right) .
$$


Similarly, if $\gamma \rightarrow-\infty$, then we have

$\operatorname{SIFWM}^{T}{ }_{\omega, g} S S(\alpha)=\left(\frac{\min \left(\mu_{\alpha}\right)}{\min \left(\mu_{\alpha}\right)+\min \left(1-\mu_{\alpha}\right)}, \frac{\min \left(v_{\alpha}\right)}{\min \left(v_{\alpha}\right)+\min \left(1-v_{\alpha}\right)}\right)$,

and if $\gamma \rightarrow 0$, then we get

$$
\begin{aligned}
& \operatorname{SIFWM}^{T, g_{0} S}(\alpha) \\
& \quad=\left(\frac{\prod_{j=1}^{n} \mu_{\alpha_{j}}^{\omega_{j}}}{\prod_{j=1}^{n}\left(1-\mu_{\alpha_{j}}\right)^{\omega_{j}}+\prod_{j=1}^{n} \mu_{\alpha_{j}}^{\omega_{j}}}, \frac{\prod_{j=1}^{n} v_{\alpha_{j}}^{\omega_{j}}}{\prod_{j=1}^{n}\left(1-v_{\alpha_{j}}\right)^{\omega_{j}}+\prod_{j=1}^{n} v_{\alpha_{j}}^{\omega_{j}}}\right) .
\end{aligned}
$$

(3) If $g(t)=g_{\gamma}^{D}(t)$, then the SIFWM ${ }^{T_{\omega, g}}$ operator is reduced to the following case:

$$
\begin{aligned}
& \operatorname{SIFWM}^{T_{\omega, g_{\gamma}}}(\alpha) \\
& \quad=\left(\frac{T_{\omega, g_{\gamma}^{D}}\left(\mu_{\alpha}\right)}{T_{\omega, g_{\gamma} D}\left(\mu_{\alpha}\right)+T_{\omega, g_{\gamma} D}\left(1-\mu_{\alpha}\right)}, \frac{T_{\omega, g_{\gamma}^{D}}\left(v_{\alpha}\right)}{T_{\omega, g_{\gamma} D}\left(v_{\alpha}\right)+T_{\omega, g_{\gamma} D}\left(1-v_{\alpha}\right)}\right) .
\end{aligned}
$$

In particular, if $\gamma=1$, then the $\operatorname{SIFWM}^{T_{\omega, g}}$ operator is reduced to the following case:

$$
\begin{aligned}
& \operatorname{SIFWM}^{T, g_{1}^{D}}(\alpha) \\
& \quad=\left(\frac{T_{\omega, g_{1}^{D}}\left(\mu_{\alpha}\right)}{T_{\omega, g_{1}}\left(\mu_{\alpha}\right)+T_{\omega, g_{1}^{D}}\left(1-\mu_{\alpha}\right)}, \frac{T_{\omega, g_{1}^{D}}\left(v_{\alpha}\right)}{T_{\omega, g_{1}^{D}}\left(v_{\alpha}\right)+T_{\omega, g_{1}^{D}}\left(1-v_{\alpha}\right)}\right),
\end{aligned}
$$

where $T_{\omega, g_{1} D}(x)=\left(\sum_{j=1}^{n} \omega_{j} x_{j}^{-1}\right)^{-1}$. Now, we consider the case for $\gamma \rightarrow \infty$. Notice that

$$
\lim _{\gamma \rightarrow \infty}\left(x_{j}^{-1}-1\right)^{\gamma}= \begin{cases}\infty, & x_{j}<\frac{1}{2} \\ 1, & x_{j}=\frac{1}{2} \\ 0, & x_{j}>\frac{1}{2}\end{cases}
$$

For $\lim _{\gamma \rightarrow \infty}\left(\sum_{j=1}^{n} \omega_{j}\left(x_{j}^{-1}-1\right)^{\gamma}\right)^{\frac{1}{\gamma}}$, taking $x_{k}=\min \left\{x_{1}, x_{2}, \ldots, x_{n}\right\}$ with $k \in$ $\{1, \ldots, n\}$, then we have the following three cases:

Case 1. $x_{k}<\frac{1}{2}$, i.e. $x_{k}^{-1}-1>1$. Then it follows from L'Hôpital's rule that

$$
\lim _{\gamma \rightarrow \infty}\left(\sum_{j=1}^{n} \omega_{j}\left(x_{j}^{-1}-1\right)^{\gamma}\right)^{\frac{1}{\gamma}}=\lim _{\gamma \rightarrow \infty} e^{\frac{\ln \left(\sum_{j=1}^{n} \omega_{j}\left(x_{j}^{-1}-1\right)^{\gamma}\right)}{\gamma}}
$$




$$
\begin{aligned}
& =\lim _{\gamma \rightarrow \infty} e^{\frac{\sum_{j=1}^{n} \omega_{j}\left(x_{j}^{-1}-1\right)^{\gamma} \ln \left(x_{j}^{-1}-1\right)}{\sum_{j=1}^{n} \omega_{j}\left(x_{j}^{-1}-1\right)^{\gamma}}}=\lim _{\gamma \rightarrow \infty} e^{\frac{\sum_{j=1}^{n} \omega_{j}\left(\frac{x_{j}^{-1}-1}{x_{k}^{-1}-1}\right)^{\gamma} \ln \left(x_{j}^{-1}-1\right)}{\sum_{j=1}^{n} \omega_{j}\left(\frac{x_{j}^{-1}-1}{x_{k}^{-1}-1}\right)^{\gamma}}} \\
& =e^{\ln \left(x_{k}^{-1}-1\right)}=x_{k}^{-1}-1 .
\end{aligned}
$$

Thus $\lim _{\gamma \rightarrow \infty} T_{\omega, g_{\gamma}^{D}}(x)=x_{k}$.

Case 2. $x_{k}=\frac{1}{2}$, i.e. $x_{k}^{-1}-1=1$. Then it follows from L'Hôpital's rule that $\lim _{\gamma \rightarrow \infty}\left(\sum_{j=1}^{n} \omega_{j}\left(x_{j}^{-1}-1\right)^{\gamma}\right)^{\frac{1}{\gamma}}=\lim _{\gamma \rightarrow \infty} e^{\frac{\ln \left(\sum_{j=1}^{n} \omega_{j}\left(x_{j}^{-1}-1\right)^{\gamma}\right)}{\gamma}}=1$ thus $\lim _{\gamma \rightarrow \infty} T_{\omega, g_{\gamma} D}(x)=\frac{1}{2}$.

Case 3. $x_{k}>\frac{1}{2}$, i.e. $x_{k}^{-1}-1<1$. Then it is similar to Case 1 that $\lim _{\gamma \rightarrow \infty}\left(\sum_{j=1}^{n} \omega_{j}\left(x_{j}^{-1}-1\right)^{\gamma}\right)^{\frac{1}{\gamma}}=x_{k}^{-1}-1$ thus $\lim _{\gamma \rightarrow \infty} T_{\omega, g_{\gamma}}(x)=x_{k}$.

All in all, we have $\lim _{\gamma \rightarrow \infty} T_{\omega, g_{\gamma}^{D}}(x)=x_{k}$, which yields that

$$
\operatorname{SIFWM}_{\omega, g_{\infty}}^{T}(\alpha)=\left(\frac{\min \left(\mu_{\alpha}\right)}{\min \left(\mu_{\alpha}\right)+\min \left(1-\mu_{\alpha}\right)}, \frac{\min \left(v_{\alpha}\right)}{\min \left(v_{\alpha}\right)+\min \left(1-v_{\alpha}\right)}\right) .
$$

Similarly, if $\gamma \rightarrow 0$, then we obtain

$$
\begin{aligned}
& \operatorname{SIFWM}^{T, g_{0}^{D}}(\alpha) \\
& \quad=\left(\frac{\prod_{j=1}^{n} \mu_{\alpha_{j}}^{\omega_{j}}}{\prod_{j=1}^{n}\left(1-\mu_{\alpha_{j}}\right)^{\omega_{j}}+\prod_{j=1}^{n} \mu_{\alpha_{j}}^{\omega_{j}}}, \frac{\prod_{j=1}^{n} v_{\alpha_{j}}^{\omega_{j}}}{\prod_{j=1}^{n}\left(1-v_{\alpha_{j}}\right)^{\omega_{j}}+\prod_{j=1}^{n} \nu_{\alpha_{j}}^{\omega_{j}}}\right) .
\end{aligned}
$$

3.2. Symmetric Intuitionistic Fuzzy Weighted Mean Operators w.r.t. Weighted Archimedean t-Conorms

Lemma 3.4. Let $\alpha_{j}(j=1,2, \ldots, n)$ be a collection of IFNs and $S_{\omega, g}$ be the dual of a weighted Archimedean $t$-norm $T_{\omega, g}$ with an additive generator $g$ w.r.t. standard negation $n(x)=1-x$. Then 


$$
\left(\frac{S_{\omega, g}\left(\mu_{\alpha}\right)}{S_{\omega, g}\left(\mu_{\alpha}\right)+S_{\omega, g}\left(1-\mu_{\alpha}\right)}, \frac{S_{\omega, g}\left(v_{\alpha}\right)}{S_{\omega, g}\left(v_{\alpha}\right)+S_{\omega, g}\left(1-v_{\alpha}\right)}\right)
$$

is an IFN.

Proof. Since $\alpha_{j}(j=1,2, \ldots, n)$ are IFNs, we have $\mu_{\alpha_{j}}+v_{\alpha_{j}} \leqslant 1$, i.e. $v_{\alpha_{j}} \leqslant 1-\mu_{\alpha_{j}}$. The antitonicity of $g$ leads that $\omega_{j} g\left(1-\mu_{\alpha_{j}}\right) \leqslant \omega_{j} g\left(v_{\alpha_{j}}\right)$, and hence $S_{\omega, g}\left(v_{\alpha}\right) \leqslant S_{\omega, g}(1-$ $\left.\mu_{\alpha}\right)$. Thus we get $\frac{S_{\omega, g}\left(\mu_{\alpha}\right)}{S_{\omega, g}\left(\mu_{\alpha}\right)+S_{\omega, g}\left(1-\mu_{\alpha}\right)} \leqslant \frac{S_{\omega, g}\left(\mu_{\alpha}\right)}{S_{\omega, g}\left(\mu_{\alpha}\right)+S_{\omega, g}\left(v_{\alpha}\right)}$. Similarly, $\frac{S_{\omega, g}\left(v_{\alpha}\right)}{S_{\omega, g}\left(v_{\alpha}\right)+S_{\omega, g}\left(1-v_{\alpha}\right)} \leqslant$ $\frac{S_{\omega, g}\left(v_{\alpha}\right)}{S_{\omega, g}\left(v_{\alpha}\right)+S_{\omega, g}\left(\mu_{\alpha}\right)}$. Thus it yields that $\frac{S_{\omega, g}\left(\mu_{\alpha}\right)}{S_{\omega, g}\left(\mu_{\alpha}\right)+S_{\omega, g}\left(1-\mu_{\alpha}\right)}+\frac{S_{\omega, g}\left(v_{\alpha}\right)}{S_{\omega, g}\left(v_{\alpha}\right)+S_{\omega, g}\left(1-v_{\alpha}\right)} \leqslant 1$, that is, (16) is an IFN.

Definition 3.5. Let $\alpha_{j}(j=1,2, \ldots, n)$ be a collection of IFNs, $S_{\omega, g}$ be the dual of a weighted Archimedean $t$-norm $T_{\omega, g}$ with an additive generator $g$ w.r.t. standard negation $n(x)=1-x$ and $\operatorname{SIFWM}^{S_{\omega, g}}: \mathcal{I} \mathcal{F} \mathcal{N}^{n} \rightarrow \mathcal{I} \mathcal{F} \mathcal{N}$, if

$$
\operatorname{SIFWM}^{S_{\omega, g}}(\alpha)=\left(\frac{S_{\omega, g}\left(\mu_{\alpha}\right)}{S_{\omega, g}\left(\mu_{\alpha}\right)+S_{\omega, g}\left(1-\mu_{\alpha}\right)}, \frac{S_{\omega, g}\left(v_{\alpha}\right)}{S_{\omega, g}\left(v_{\alpha}\right)+S_{\omega, g}\left(1-v_{\alpha}\right)}\right),
$$

then SIFWM $^{S_{\omega, g}}$ is called a symmetric intuitionistic fuzzy weighted mean (SIFWM ${ }^{S_{\omega, g}}$ ) operator of dimension $n$ w.r.t. $S_{\omega, g}$.

Especially, if $v_{\alpha_{j}}=1-\mu_{\alpha_{j}}$ for all $j=1,2, \ldots, n$, that is, all $\alpha_{j}$ are reduced to $\mu_{\alpha_{j}}$, respectively, then Eq. (17) is reduced to the following form:

$$
\operatorname{SIFWM}^{S_{\omega, g}}(\alpha)=\left(\frac{S_{\omega, g}\left(\mu_{\alpha}\right)}{S_{\omega, g}\left(1-\mu_{\alpha}\right)+S_{\omega, g}\left(\mu_{\alpha}\right)}, 1-\frac{S_{\omega, g}\left(\mu_{\alpha}\right)}{S_{\omega, g}\left(1-\mu_{\alpha}\right)+S_{\omega, g}\left(\mu_{\alpha}\right)}\right),
$$

which becomes both a symmetric sum operator of dimension $n$ (Beliakov et al., 2007) and a weighted $t$-norm-based aggregation operator with $H(x, y)=\frac{y}{1-x+y}$ (Calvo and Mesiar, 2003) to aggregate fuzzy information.

Proposition 3.6. Let $\alpha_{j}, \beta_{j}(j=1,2, \ldots, n)$ be two collections of IFNs with $\alpha=$ $\left(\alpha_{1}, \alpha_{2}, \ldots, \alpha_{n}\right), \beta=\left(\beta_{1}, \beta_{2}, \ldots, \beta_{n}\right)$, respectively, and $S_{\omega, g}$ be the dual of a weighted Archimedean $t$-norm $T_{\omega, g}$ with an additive generator $g$ w.r.t. standard negation $n(x)=$ $1-x$.

(1) If all $\alpha_{j}$ are equal, i.e. $\alpha_{j}=\delta=\left(\mu_{\delta}, v_{\delta}\right)$, for all $j$, then $\operatorname{SIFWM}^{S_{\omega, g}}(\alpha)=\delta$;

(2) If $\alpha_{j} \leqslant \beta_{j}$ for all $j$, then $\operatorname{SIFWM}^{S_{\omega, g}}(\alpha) \leqslant \operatorname{SIFWM}^{S_{\omega, g}}(\beta)$;

(3) Let $\alpha^{-}=\left(\min \left(\mu_{\alpha}\right), \max \left(v_{\alpha}\right)\right)$ and $\alpha^{+}=\left(\max \left(\mu_{\alpha}\right), \min \left(v_{\alpha_{j}}\right)\right)$, then $\alpha^{-} \leqslant$ $\operatorname{SIFWM}^{S_{\omega, g}}(\alpha) \leqslant \alpha^{+}$.

Proof. It can be proved in a similar way as in Proposition 3.3.

Next, we assign the additive generator $g$ to different forms, some specific symmetric intuitionistic fuzzy aggregation operators can be obtained as follows: 
1. If $g(t)=g_{\gamma}^{H}(1-t)$, then the $\operatorname{SIFWM}^{S_{\omega, g}}$ operator is reduced to the following form: $\operatorname{SIFWM}^{S_{\omega, g_{\gamma}^{H}}^{H}}(\alpha)$

$$
=\left(\frac{S_{\omega, g_{\gamma}^{H}}\left(\mu_{\alpha}\right)}{S_{\omega, g_{\gamma}^{H}}\left(1-\mu_{\alpha}\right)+S_{\omega, g_{\gamma}^{H}}\left(\mu_{\alpha}\right)}, \frac{S_{\omega, g_{\gamma}^{H}}\left(v_{\alpha}\right)}{S_{\omega, g_{\gamma}^{H}}\left(1-v_{\alpha}\right)+S_{\omega, g_{\gamma}^{H}}\left(v_{\alpha}\right)}\right),
$$

where $S_{\omega, g_{\gamma} H}(x)=\frac{\prod_{j=1}^{n}\left(\frac{\gamma}{1-x_{j}}+1-\gamma\right)^{\omega_{j}}-1}{\prod_{j=1}^{n}\left(\frac{\gamma}{1-x_{j}}+1-\gamma\right)^{\omega} j+\gamma-1}, \gamma>0$.

Especially, if $\gamma=1$, i.e. $g_{1}^{H}(t)=-\ln (1-t)$, then the SIFWM ${ }^{S_{\omega, g_{1}^{H}}}$ operator with $S_{\omega, g_{1}^{H}}(x)=1-\prod_{j=1}^{n}\left(1-x_{j}\right)^{\omega_{j}}$ is the symmetric form of intuitionistic fuzzy weighted averaging (IFWA) operator defined by $\mathrm{Xu}(2007)$; if $\gamma=2$, i.e. $g_{2}^{H}(t)=$ $\ln \left(\frac{1+t}{1-t}\right)$, then the SIFWM ${ }^{S_{, g_{2}}^{H}}$ operator with $S_{\omega, g_{2}^{H}}(x)=\frac{\prod_{j=1}^{n}\left(\frac{1+x_{j}}{1-x_{j}}\right)^{\omega_{j}}-1}{\prod_{j=1}^{n}\left(\frac{1+x_{j}}{1-x_{j}}\right)^{\omega_{j}}+1}$ is the symmetric form of IFWG operator based on Einstein $t$-norm and $t$-conorm defined by Wang and Liu (2012); if $\gamma \rightarrow \infty$, then it is similar to the proof of $\lim _{\gamma \rightarrow \infty} T_{\omega, g_{\gamma}^{H}}(x)$ that

$$
\lim _{\gamma \rightarrow \infty} S_{\omega, g_{\gamma}^{H}}(x)=\frac{\prod_{j=1}^{n} x_{j}^{\omega_{j}}}{\prod_{j=1}^{n} x_{j}^{\omega_{j}}+\prod_{j=1}^{n}\left(1-x_{j}\right)^{\omega_{j}}},
$$

and hence

$$
\begin{aligned}
& \operatorname{SIFWM}_{\omega, g \infty}^{S_{\infty}}(\alpha) \\
& \quad=\left(\frac{\prod_{j=1}^{n} \mu_{\alpha_{j}}^{\omega_{j}}}{\prod_{j=1}^{n}\left(1-\mu_{\alpha_{j}}\right)^{\omega_{j}}+\prod_{j=1}^{n} \mu_{\alpha_{j}}^{\omega_{j}}}, \frac{\prod_{j=1}^{n} v_{\alpha_{j}}^{\omega_{j}}}{\prod_{j=1}^{n}\left(1-v_{\alpha_{j}}\right)^{\omega_{j}}+\prod_{j=1}^{n} v_{\alpha_{j}}^{\omega_{j}}}\right) .
\end{aligned}
$$

2. If $g(t)=g_{\gamma}^{S S}(1-t)$, then the $\operatorname{SIFWM}^{S_{\omega, g}}$ operator is reduced to the following form:

$$
\begin{aligned}
& \operatorname{SIFWM}^{{ }^{{ }_{\omega, g_{\gamma}} S}(\alpha)} \\
& \quad=\left(\frac{S_{\omega, g_{\gamma} S}\left(\mu_{\alpha}\right)}{S_{\omega, g_{\gamma} S S}\left(\mu_{\alpha}\right)+S_{\omega, g_{\gamma} S S}\left(1-\mu_{\alpha}\right)}, \frac{S_{\omega, g_{\gamma} S S}\left(v_{\alpha}\right)}{S_{\omega, g_{\gamma} S S}\left(v_{\alpha}\right)+S_{\omega, g_{\gamma} S S}\left(1-v_{\alpha}\right)}\right),
\end{aligned}
$$

where $S_{\omega, g_{\gamma}^{S S}}(x)=1-\left(\sum_{j=1}^{n} \omega_{j}\left(1-x_{j}\right)^{\gamma}\right)^{\frac{1}{\gamma}}, \gamma \neq 0$. In particular, it is similar to the proofs of $\operatorname{SIFWM}^{T, g_{\infty} S S}(\alpha), \operatorname{SIFWM}^{T, g_{-\infty} S}(\alpha){\text { and } \operatorname{SIFWM}^{T, g_{0} S}(\alpha) \text { that }}^{T}$

$$
\begin{aligned}
& \operatorname{SIFWM}^{S_{\omega, g} S S}(\alpha)=\left(\frac{\min \left(\mu_{\alpha}\right)}{\min \left(\mu_{\alpha}\right)+\min \left(1-\mu_{\alpha}\right)}, \frac{\min \left(v_{\alpha}\right)}{\min \left(v_{\alpha}\right)+\min \left(1-v_{\alpha}\right)}\right), \\
& \operatorname{SIFWM}^{S_{\omega, g} S S}(\alpha)=\left(\frac{\max \left(\mu_{\alpha}\right)}{\max \left(\mu_{\alpha}\right)+\max \left(1-\mu_{\alpha}\right)}, \frac{\max \left(v_{\alpha}\right)}{\max \left(v_{\alpha}\right)+\max \left(1-v_{\alpha}\right)}\right),
\end{aligned}
$$




$$
\operatorname{SIFWM}^{{ }_{\omega, g_{0}}^{S S}}(\alpha)=\left(\frac{\prod_{j=1}^{n} \mu_{\alpha_{j}}^{\omega_{j}}}{\prod_{j=1}^{n}\left(1-\mu_{\alpha_{j}}\right)^{\omega_{j}}+\prod_{j=1}^{n} \mu_{\alpha_{j}}^{\omega_{j}}}, \frac{\prod_{j=1}^{n} v_{\alpha_{j}}^{\omega_{j}}}{\prod_{j=1}^{n}\left(1-v_{\alpha_{j}}\right)^{\omega_{j}}+\prod_{j=1}^{n} \nu_{\alpha_{j}}^{\omega_{j}}}\right)
$$

(3) If $g(t)=g_{\gamma}^{D}(1-t)$, then the $\operatorname{SIFWM}^{S_{\omega, g}}$ operator is reduced to the following form:

$$
\begin{aligned}
& \operatorname{SIFWM}^{S_{\omega, g_{\gamma}}^{D}}(\alpha) \\
& \quad=\left(\frac{S_{\omega, g_{\gamma}^{D}}\left(\mu_{\alpha}\right)}{S_{\omega, g_{\gamma}^{D}}\left(\mu_{\alpha}\right)+S_{\omega, g_{\gamma}^{D}}\left(1-\mu_{\alpha}\right)}, \frac{S_{\omega, g_{\gamma}^{D}}\left(v_{\alpha}\right)}{S_{\omega, g_{\gamma}^{D}}\left(v_{\alpha}\right)+S_{\omega, g_{\gamma}^{D}}\left(1-v_{\alpha}\right)}\right),
\end{aligned}
$$

where $S_{\omega, g_{\gamma} D}(x)=\frac{\left(\sum_{j=1}^{n} \omega_{j}\left(\frac{x_{j}}{1-x_{j}}\right)^{\gamma}\right)^{\frac{1}{\gamma}}}{\left(\sum_{j=1}^{n} \omega_{j}\left(\frac{x_{j}}{1-x_{j}}\right)^{\gamma}\right)^{\frac{1}{\gamma}}+1}, \gamma>0$.

Particularly, if $\gamma=1$, then the $\operatorname{SIFWM}^{S_{\omega, g}}$ operator is reduced to the following form:

$$
\begin{aligned}
& \operatorname{SIFWM}_{\omega, g_{1}}^{S_{\omega}}(\alpha) \\
& \quad=\left(\frac{S_{\omega, g_{1}^{D}}\left(\mu_{\alpha}\right)}{S_{\omega, g_{1} D}\left(\mu_{\alpha}\right)+S_{\omega, g_{1}^{D}}\left(1-\mu_{\alpha}\right)}, \frac{S_{\omega, g_{1}^{D}}\left(v_{\alpha}\right)}{S_{\omega, g_{1} D}\left(v_{\alpha}\right)+S_{\omega, g_{1}^{D}}\left(1-v_{\alpha}\right)}\right),
\end{aligned}
$$

where $S_{\omega, g_{1}^{D}}(x)=\frac{\sum_{j=1}^{n} \omega_{j} \frac{x_{j}}{1-x_{j}}}{\sum_{j=1}^{n} \omega_{j} \frac{1}{1-x_{j}}}$; it is similar to the proofs of $\operatorname{SIFWM}^{T} \operatorname{Isg}_{\infty}(\alpha)$ and $\operatorname{SIFWM}^{T \omega, g_{0}^{D}}(\alpha)$ that

$$
\operatorname{SIFWM}^{S}{ }_{\omega, g_{\infty} D}(\alpha)=\left(\frac{\max \left(\mu_{\alpha}\right)}{\max \left(\mu_{\alpha}\right)+\max \left(1-\mu_{\alpha}\right)}, \frac{\max \left(v_{\alpha}\right)}{\max \left(v_{\alpha}\right)+\max \left(1-v_{\alpha}\right)}\right),
$$

$$
\begin{aligned}
& \operatorname{SIFWM}^{S_{,, g_{0}}}(\alpha) \\
& \quad=\left(\frac{\prod_{j=1}^{n} \mu_{\alpha_{j}}^{\omega_{j}}}{\prod_{j=1}^{n}\left(1-\mu_{\alpha_{j}}\right)^{\omega_{j}}+\prod_{j=1}^{n} \mu_{\alpha_{j}}^{\omega_{j}}}, \frac{\prod_{j=1}^{n} v_{\alpha_{j}}^{\omega_{j}}}{\prod_{j=1}^{n}\left(1-v_{\alpha_{j}}\right)^{\omega_{j}}+\prod_{j=1}^{n} v_{\alpha_{j}}^{\omega_{j}}}\right) .
\end{aligned}
$$

All in all, we have the following conclusion:

\section{Corollary 3.7.}

(1) $\operatorname{SIFWM}^{T \omega, g_{1}^{H}}=\operatorname{SIFWM}^{T, g_{\infty}^{H}}=\operatorname{SIFWM}^{T, g_{0}^{S S}}=\operatorname{SIFWM}^{T \omega, g_{0}^{D}}=\operatorname{SIFWM}^{T_{\omega, g_{\infty}^{H}}^{H}}=$ SIFWM $^{S_{\omega, g_{1}^{H}}^{H}}=$ SIFWM $^{S_{\omega, g} S S}=$ SIFWM $^{{ }_{\omega, g_{0}}}$;

(2) $\operatorname{SIFWM}^{S}{ }_{\omega, g_{\infty} S S}=\operatorname{SIFWM}^{T, g_{-\infty}}=\operatorname{SIFWM}^{T, g_{\infty}}$;

(3) $\operatorname{SIFWM}^{T}{ }^{T}, g_{\infty}^{S S}=\operatorname{SIFWM}^{S}{ }^{\omega, g} g_{-\infty} S=\operatorname{SIFWM}^{S_{\omega, g} D}$;

(4) $\operatorname{SIFWM}^{T \omega, g_{0}^{H}}=\operatorname{SIFWM}^{T \omega, g_{-1} S S}$. 
The above corollary indicates that the operators SIFWM ${ }^{T}{ }^{2} g_{\gamma}^{S S}$ and $\operatorname{SIFWM}^{S, g_{\gamma}^{S S}}$ can well reflect the variations of the other operators. Furthermore, since SIFWM ${ }^{T} g_{\gamma}^{S S}$ and SIFWM ${ }^{S}{ }_{\omega, g_{\gamma} S S}$ are dual, we always use the operator SIFWM $^{T, g_{\gamma} S}$ to neutrally aggregate the IFNs and the Eqs. (10), (11) and (12) can be considered as the cases when the decision maker is optimistic, pessimistic or impartial. With respect to the existing symmetrical intuitionistic fuzzy aggregation operators (Beliakov et al., 2011; Liao and Xu, 2015; Xia and $\mathrm{Xu}, 2012$ ), the proposed aggregation operators based on weighted Archimedean $t$ norm and $t$-conorm possess the following advantages:

- these existing operators (Beliakov et al., 2011; Liao and Xu, 2015; Xia and Xu, 2012) can only treat membership and non-membership information fairly, and provide a single choice for the decision maker; the proposed ones can not only treat membership and non-membership information fairly but also provide more choices for the decision maker;

- the existing operator (Liao and $\mathrm{Xu}, 2015$ ) can not reduce to the corresponding fuzzy one; the proposed ones can be considered as generalizations of the existing aggregation operators in fuzzy cases;

- the existing operator (Xia and $\mathrm{Xu}, 2012)$ is not suitable for dealing with IFNs $(1,0)$ or $(0,1)$; the proposed operators can solve the case.

\section{The Relationships Among the Proposed Aggregation Operators and the Existing One}

The following lemma is obvious but useful to investigate the relationships among the proposed aggregation operators and the existing ones:

Lemma 4.1. Let $x, y, \omega_{i}, x_{i} \in[0,1], \lambda>0$ such that $\sum_{i=1}^{n} \omega_{i}=1$, it holds that

(1) Ma and $X u$ (2016) if $y \leqslant x$, then $y \leqslant \frac{y}{1-x+y} \leqslant x$ and $y \leqslant \frac{x}{1+x-y} \leqslant x$;

(2) if $g$ is convex (concave), then $g\left(\sum_{i=1}^{n} \omega_{i} x_{i}\right) \leqslant(\geqslant) \sum_{i=1}^{n} \omega_{i} g\left(x_{i}\right)$, the equality holds if and only if $x_{1}=x_{2}=\cdots=x_{n}$ or $g$ is linear.

The relationships among $\operatorname{IFWM}^{T_{\omega, g}}, \mathrm{IFWA}^{T_{\omega, g}}$ and $\mathrm{SIFWM}^{T_{\omega, g}}$ can be shown as follows:

Proposition 4.2. Let $\alpha_{j}(j=1,2, \ldots, n)$ be a collection of IFNs and $T_{\omega, g}$ be a weighted Archimedean $t$-norm $T_{\omega, g}$ with an additive generator $g$.

(1) If $g$ is concave, then $\operatorname{IFWM}^{T_{\omega, g}}(\alpha) \leqslant \operatorname{SIFWM}^{T_{\omega, g}}(\alpha) \leqslant \operatorname{IFWA}^{T_{\omega, g}}(\alpha)$;

(2) If $g$ is convex, then $\operatorname{IFWA}^{T_{\omega, g}}(\alpha) \leqslant \operatorname{SIFWM}^{T_{\omega, g}}(\alpha) \leqslant \operatorname{IFWM}^{T_{\omega, g}}(\alpha)$;

(3) If $g$ has at least one inflection point, then the inequality varies with concavityconvexity of $g$.

Proof. We only prove (1), and (2), (3) can be proven in a similar way. 
Since $g$ is concave, i.e. $g^{-1}$ is convex, it follows from Lemma 4.1(2) that $g^{-1}\left(\sum_{i=1}^{n} \omega_{i} g\left(\mu_{\alpha_{i}}\right)\right)+g^{-1}\left(\sum_{i=1}^{n} \omega_{i} g\left(1-\mu_{\alpha_{i}}\right)\right) \leqslant \sum_{i=1}^{n} \omega_{i} \mu_{\alpha_{i}}+\sum_{i=1}^{n} \omega_{i}\left(1-\mu_{\alpha_{i}}\right)=$ $\sum_{i=1}^{n} \omega_{i}=1$, and hence $g^{-1}\left(\sum_{i=1}^{n} \omega_{i} g\left(\mu_{\alpha_{i}}\right)\right) \leqslant 1-g^{-1}\left(\sum_{i=1}^{n} \omega_{i} g\left(1-\mu_{\alpha_{i}}\right)\right)$, i.e. $T_{\omega, g}\left(\mu_{\alpha}\right) \leqslant 1-T_{\omega, g}\left(1-\mu_{\alpha}\right)$. Using Lemma 4.1(1), we get $T_{\omega, g}\left(\mu_{\alpha}\right) \leqslant \frac{T_{\omega, g}\left(\mu_{\alpha}\right)}{T_{\omega, g}\left(\mu_{\alpha}\right)+T_{\omega, g}\left(1-\mu_{\alpha}\right)}$ $\leqslant 1-T_{\omega, g}\left(1-\mu_{\alpha}\right)$. In a similar way, it yields that $T_{\omega, g}\left(v_{\alpha}\right) \leqslant \frac{T_{\omega, g}\left(v_{\alpha}\right)}{T_{\omega, g}\left(v_{\alpha}\right)+T_{\omega, g}\left(1-v_{\alpha}\right)} \leqslant$ $1-T_{\omega, g}\left(1-v_{\alpha}\right)$. Thus, $\operatorname{IFWM}^{T_{\omega, g}}(\alpha) \leqslant \operatorname{SIFWM}^{T_{\omega, g}}(\alpha) \leqslant \operatorname{IFWA}^{T_{\omega, g}}(\alpha)$.

The relationships between $\operatorname{IFWM}^{S_{\omega, g}}, \operatorname{IFWA}^{S_{\omega, g}}$ and $\operatorname{SIFWM}^{S_{\omega, g}}$ can be shown as follows:

Proposition 4.3. Let $\alpha_{j}(j=1,2, \ldots, n)$ be a collection of IFNs and $S_{\omega, g}$ be the dual of a weighted Archimedean $t$-norm $T_{\omega, g}$ with an additive generator $g$ w.r.t. standard negation $n(x)=1-x$.

(1) If $g$ is concave, then $\operatorname{IFWM}^{S_{\omega, g}}(\alpha) \leqslant \operatorname{SIFWM}^{S_{\omega, g}}(\alpha) \leqslant \operatorname{IFWA}^{S_{\omega, g}}(\alpha)$;

(2) If $g$ is convex, then $\operatorname{IFWA}^{S_{\omega, g}}(\alpha) \leqslant \operatorname{SIFWM}^{S_{\omega, g}}(\alpha) \leqslant \operatorname{IFWM}^{S_{\omega, g}}(\alpha)$;

(3) If $g$ has at least one inflection point, then the inequality varies with concavityconvexity of $g$.

Proof. We only prove (1), and (2), (3) can be proven in a similar way.

Since $g$ is concave, i.e. $g^{-1}$ is convex, it follows from Lemma 4.1(2) that $g^{-1}\left(\sum_{i=1}^{n} \omega_{i} g\left(\mu_{\alpha_{i}}\right)\right)+g^{-1}\left(\sum_{i=1}^{n} \omega_{i} g\left(1-\mu_{\alpha_{i}}\right)\right) \leqslant \sum_{i=1}^{n} \omega_{i} \mu_{\alpha_{i}}+\sum_{i=1}^{n} \omega_{i}\left(1-\mu_{\alpha_{i}}\right)=$ $\sum_{i=1}^{n} \omega_{i}=1$, and hence $g^{-1}\left(\sum_{i=1}^{n} \omega_{i} g\left(\mu_{\alpha_{i}}\right)\right) \leqslant 1-g^{-1}\left(\sum_{i=1}^{n} \omega_{i} g\left(1-\mu_{\alpha_{i}}\right)\right)$, i.e. $T_{\omega, g}\left(\mu_{\alpha}\right) \leqslant 1-T_{\omega, g}\left(1-\mu_{\alpha}\right)$. By Lemma 4.1(1), we get $T_{\omega, g}\left(\mu_{\alpha}\right) \leqslant \frac{1-T_{\omega, g}\left(1-\mu_{\alpha}\right)}{2-T_{\omega, g}\left(\mu_{\alpha}\right)-T_{\omega, g}\left(1-\mu_{\alpha}\right)}$ $=\frac{S_{\omega, g}\left(\mu_{\alpha}\right)}{S_{\omega, g}\left(\mu_{\alpha}\right)+S_{\omega, g}\left(1-\mu_{\alpha}\right)} \leqslant 1-T_{\omega, g}\left(1-\mu_{\alpha}\right)$. In a similar way, we have $T_{\omega, g}\left(v_{\alpha}\right) \leqslant$ $\frac{S_{\omega, g}\left(v_{\alpha}\right)}{S_{\omega, g}\left(v_{\alpha}\right)+S_{\omega, g}\left(1-v_{\alpha}\right)} \leqslant 1-T_{\omega, g}\left(1-v_{\alpha}\right)$. Thus $I F W M^{S_{\omega, g}}(\alpha) \leqslant S I F W M^{S_{\omega, g}}(\alpha) \leqslant$ IF W $A^{S_{\omega, g}}(\alpha)$.

\section{An Approach to Intuitionistic Fuzzy Multi-Criteria Decision Making}

For a multi-criteria decision making under intuitionistic fuzzy environment, let $x=$ $\left\{x_{1}, x_{2}, \ldots, x_{m}\right\}$ be a set of alternatives to be selected, and $C=\left\{C_{1}, C_{2}, \ldots, C_{n}\right\}$ be a set of criteria to be evaluated. To evaluate the performance of the alternative $x_{i}$ under the criterion $C_{j}$, the decision maker is required to provide not only the information that the alternative $x_{i}$ satisfies the criterion $C_{j}$, but also the information that the alternative $x_{i}$ does not satisfy the criterion $C_{j}$. This two part information can be expressed by $\mu_{i j}$ and $v_{i j}$ which denote the degrees that the alternative $x_{i}$ satisfies the criterion $C_{j}$ and does not satisfy the criterion $C_{j}$, then the performance of the alternative $x_{i}$ under the criteria $C_{j}$ can be expressed by an IFN $\alpha_{i j}$ with the condition that $0 \leqslant \mu_{i j}, v_{i j} \leqslant 1$ and $0 \leqslant \mu_{i j}+v_{i j} \leqslant 1$. When all the performances of the alternatives are provided, the intuitionistic fuzzy decision matrix $D=\left(\alpha_{i j}\right)_{m \times n}=\left(\left(\mu_{i j}, v_{i j}\right)\right)_{m \times n}$ can be constructed. To obtain the ranking of the alternatives, the following steps are given: 
Table 1

Intuitionistic fuzzy decision matrix $D$.

\begin{tabular}{lllll}
\hline & $C_{1}$ & $C_{2}$ & $C_{3}$ & $C_{4}$ \\
\hline$x_{1}$ & $(0.60,0.18)$ & $(0.24,0.44)$ & $(0.10,0.54)$ & $(0.45,0.23)$ \\
$x_{2}$ & $(0.41,0.25)$ & $(0.49,0.09)$ & $(0.10,0.39)$ & $(0.52,0.45)$ \\
$x_{3}$ & $(0.62,0.18)$ & $(0.67,0.28)$ & $(0.36,0.42)$ & $(0.12,0.67)$ \\
$x_{4}$ & $(0.21,0.58)$ & $(0.76,0.22)$ & $(0.48,0.34)$ & $(0.15,0.53)$ \\
$x_{5}$ & $(0.38,0.19)$ & $(0.65,0.32)$ & $(0.06,0.29)$ & $(0.24,0.39)$ \\
$x_{6}$ & $(0.56,0.12)$ & $(0.50,0.41)$ & $(0.21,0.07)$ & $(0.06,0.28)$ \\
\hline
\end{tabular}

(1) Transform the intuitionistic fuzzy decision matrix $D=\left(\left(\mu_{i j}, v_{i j}\right)\right)_{m \times n}$ into the normalized one $B=\left(\left(\beta_{i j}\right)\right)_{m \times n}$, where

$$
\beta_{i j}= \begin{cases}\alpha_{i j}, & \text { for benefit attribute } x_{i} \\ \alpha_{i j}^{c}, & \text { for cost attribute } x_{i}\end{cases}
$$

(2) Aggregate the IFNs $\beta_{i j}(j=1,2, \ldots, n)$ of the alternative $x_{i}(i=1,2, \ldots, m)$, denoted as $\beta_{i}(i=1,2, \ldots, m)$, by the proposed aggregation operators $\operatorname{SIFWM}^{T, g_{\gamma} S}$ (9).

(3) Calculate the score $s\left(\beta_{i}\right)$ of $\beta_{i}(i=1,2, \ldots, m)$ by Definition 2.6, and obtain the priority of the alternatives according to the ranking of $\beta_{i}(i=1,2, \ldots, m)$, the bigger the IFN $\beta_{i}$ is, the better the alternative $x_{i}$ is.

To illustrate the proposed methods, an example adapted from Chen (2011), Xia et al. (2012) is given as follows:

EXAMPLE 5.1. The purchasing manager in a small enterprise considers various criteria involving $C_{1}$ : financial factors (e.g. economic performance, financial stability), $C_{2}$ : performance (e.g. delivery, quality, price), $C_{3}$ : technology (e.g. manufacturing capability, design capability, ability to cope with technology changes), and $C_{4}$ : organizational culture and strategy (e.g. feeling of trust, internal and external integration of suppliers, compatibility across levels and functions of the buyer and supplier). The set of evaluative criteria is denoted by $\mathcal{C}=\left\{C_{1}, C_{2}, C_{3}, C_{4}\right\}$, whose weight vector is $\omega=(0.34,0.23,0.22,0.21)^{\top}$. There are six suppliers available, and the set of all alternatives is denoted by $X=$ $\left\{x_{1}, x_{2}, \ldots, x_{6}\right\}$. The characteristics of the suppliers $x_{i}(i=1,2, \ldots, 6)$ in terms of the criteria in $C$ are expressed by the following intuitionistic fuzzy decision matrix (see Table 1).

As it has been pointed in Xia et al. (2012) that all the criteria $C_{j}(j=1,2,3,4)$ are the benefit criteria, the IFNs of the alternatives $x_{i}(i=1,2, \ldots, 6)$ do not need normalization. Thus to obtain the alternative(s), the following steps are given:

(1) Aggregate the IFNs $\beta_{i j}(j=1,2,3,4)$ of the alternative $x_{i}(i=1,2,3,4,5,6)$, denoted as $\beta_{i}(i=1,2,3,4,5,6)$, by the $\operatorname{SIFWM}^{T}{ }_{\omega, g_{\gamma} S S}$ operator (9). 


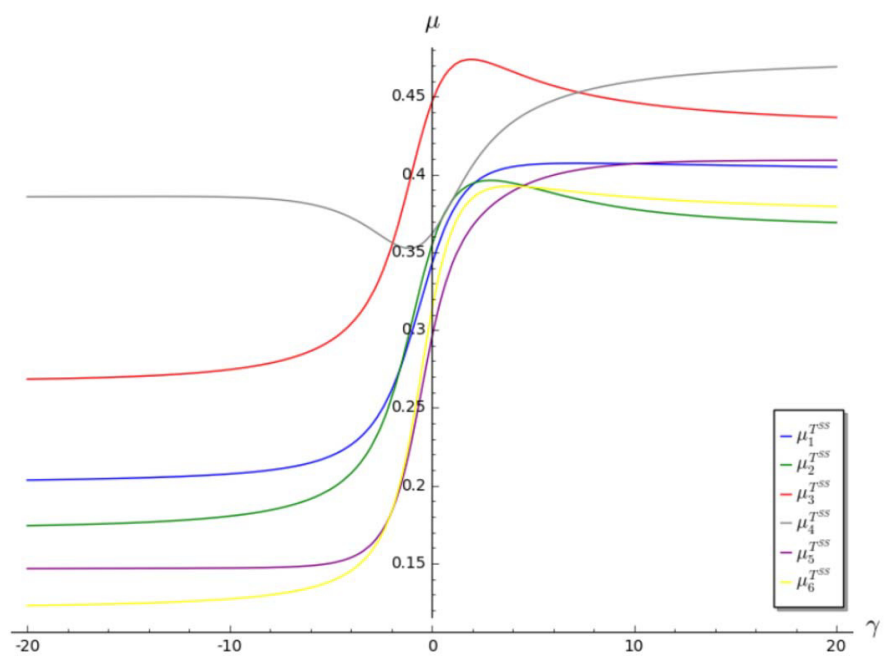

Fig. 1. Variation of the memberships of the aggregated results by SIFWM ${ }^{T} g_{\gamma}^{S S}$ operators.

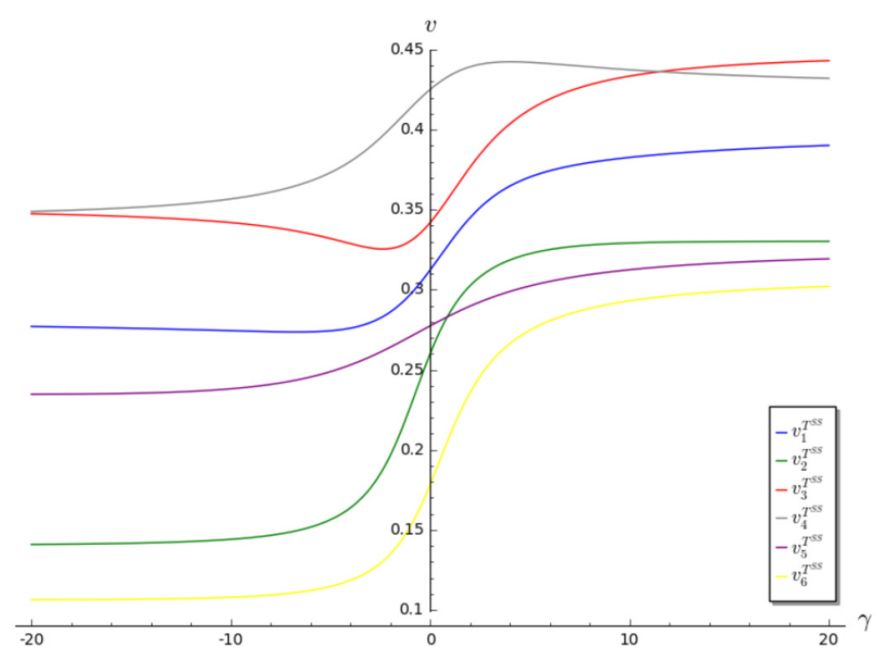

Fig. 2. Variation of the nonmemberships of the aggregated results by SIFWM ${ }^{T} g_{\gamma}^{S S}$ operators.

(2) Calculate the score $s\left(\beta_{i}\right)$ of $\beta_{i}(i=1,2,3,4,5,6)$ by item (2.2) which is shown in Figs. 1, 2 and 3.

Obviously, the aggregated results and the ranking orders of the alternatives vary with the parameter $\gamma$, that is, they can be considered as the function with $\gamma$ as its independent variable. Thus we can illustrate them by their functional images as follows:

Fig. 1 gives the variation of the memberships of the aggregated results by SIFWM ${ }^{T \omega, g_{\gamma} S}$ operators, denoted as $\mu_{i}^{T_{\omega, g_{\gamma}^{H}}}(i=1,2, \ldots, 6)$, respectively, with the parameter $\gamma$ 


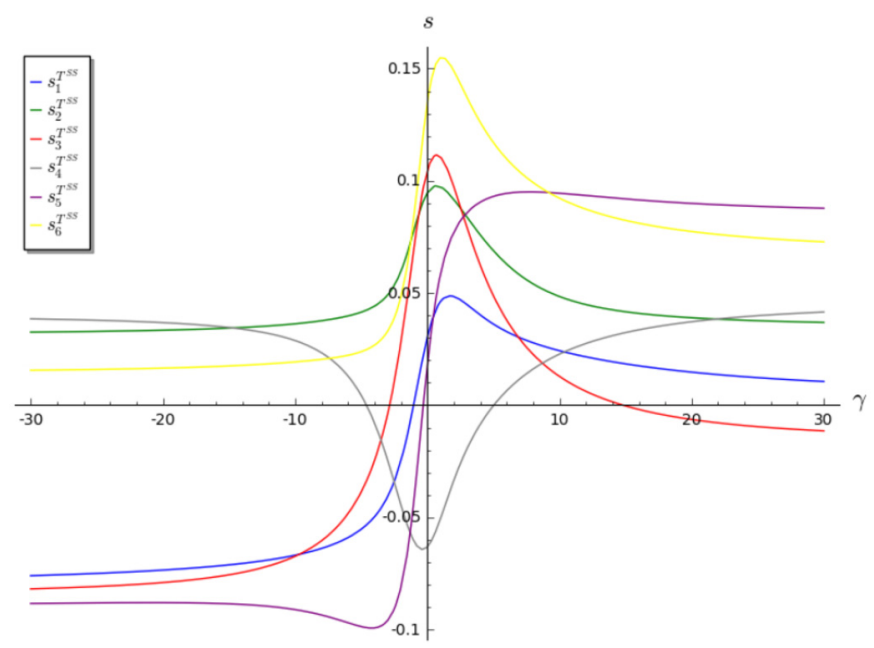

Fig. 3. Variation of the scores of the aggregated results by SIFWM ${ }^{T}{ }^{g, g_{\gamma} S}$ operators.

from -20 to 20. Particularly, when $\gamma=0$, it is the result obtained by the operator in Xia et al. (2012); when $\gamma=1$, it is the result obtained by the operator in Beliakov et al. (2011).

Fig. 2 indicates the variation of the nonmemberships of the aggregated results by SIFWM $^{T \omega, g_{\gamma} S}$ operators where the values of $\gamma$ increase from -20 to 20. Similarly, when $\gamma=0$, it is the result obtained by the operator in Xia et al. (2012); when $\gamma=1$, it is the result obtained by the operator in Beliakov et al. (2011).

Fig. 3 provides the variation of the scores of the alternatives obtained by the SIFWM ${ }^{T}, g_{\gamma}^{S S}$ operator with $\gamma$ from -30 to 30 . When $\gamma<-14.7$, that is, pessimistically, the optimal alternative is $x_{4}$; when $-14.7<\gamma<-1.2$, relatively pessimistically, optimal one is $x_{2}$; when $-1.2<\gamma<9.2$, impartially the optimal one is $x_{6}$; when $9.2<\gamma$, optimistically, the optimal one is $x_{5}$. It is obvious that the alternative $x_{5}$ varies from the worst one to the optimal one with the parameter $\gamma$ which reflects the attitude of the decision maker.

In order to compare the ranking orders with those that are provided by SIFWG operator (Liao and $\mathrm{Xu}, 2015$ ), we take $\gamma=0$, and $\gamma=1$, that is, the results obtained by the operators in Xia and Xu (2012), Beliakov et al. (2011), which are listed in Table 2.

\section{Conclusions}

Various aggregation operators have been constructed to adapt to different situations. In this paper, we proposed the SIFWM operators w.r.t. weighted Archimedean $t$-norms and $t$-conorms to neutrally deal with membership and non-membership of intuitionistic fuzzy information. Comparing the existing symmetrical operators with the proposed ones, we found that 
Table 2

Ranking orders determined by different aggregation operators.

\begin{tabular}{ll}
\hline Operator & Ranking order \\
\hline The proposed operator with $\gamma=-\infty$ & $x_{4} \succ x_{2} \succ x_{6} \succ x_{1} \succ x_{3} \succ x_{5}$ \\
The proposed operator with $\gamma=-1$ & $x_{6} \succ x_{2} \succ x_{3} \succ x_{1} \succ x_{5} \succ x_{4}$ \\
The proposed operator with $\gamma=0$ (Xia and Xu, 2012) & $x_{6} \succ x_{3} \succ x_{2} \succ x_{1} \succ x_{5} \succ x_{4}$ \\
The proposed operator with $\gamma=1$ (Beliakov et al., 2011) & $x_{6} \succ x_{3} \succ x_{2} \succ x_{5} \succ x_{1} \succ x_{4}$ \\
The proposed operator with $\gamma=\infty$ & $x_{5} \succ x_{6} \succ x_{2} \succ x_{4} \succ x_{1} \succ x_{3}$ \\
The operator provided by Liao (Liao and Xu, 2015) & $x_{6} \succ x_{2} \succ x_{3} \succ x_{1} \succ x_{5} \succ x_{4}$ \\
\hline
\end{tabular}

(1) the existing symmetrical operators in Beliakov et al. (2011), Xia and Xu (2012) are special cases of the proposed ones with constant parameters which only reflect the impartial attitude of the decision maker;

(2) the proposed symmetrical operators can not only reflect the impartial attitude of the decision maker but also the optimistic or pessimistic attitude by a parameter, which provides more choices for the decision maker in the procedure of decision making.

In the future, we will utilize the symmetrical aggregation operators in other fuzzy environments such as linguistic, bipolar, Pythagorean and intuitionistic multiplicative fuzzy environment (Alghamdi et al., 2018; Alonso et al., 2013; Ma and Xu, 2016, 2018) to investigate the consensus in group decision making problems (Del Moral et al., 2018; Dong et al., 2018; Urena et al., 2019).

Acknowledgements. The authors would like to thank the editors and the anonymous reviewers for their insightful and constructive comments and suggestions that have led to this improved version of the paper.

\section{Funding}

This research was supported by the NSF of Shandong Province (No. ZR2017MG027) and AMEP (DYSP) of Linyi University (No. LYDX2014BS017).

\section{References}

Atanassov, K. (1986). Intuitionistic fuzzy set. Fuzzy Sets and Systems, 20, 87-96.

Alghamdi, M.A., Alshehri, N.O., Akram, M. (2018). Multi-criteria decision-making methods in bipolar fuzzy environment. International Journal of Fuzzy Systems, 20, 2057-2064.

Alonso, S., Perez, I.J., Cabrerizo, F.J., Herrera-Viedma, E. (2013). A linguistic consensus model for Web 2.0 communities. Applied Soft Computing, 13, 149-157.

Beliakov, G., James, S. (2013). On extending generalized Bonferroni means to Atanassov orthopairs in decision making contexts. Fuzzy Sets and Systems, 211, 84-98.

Beliakov, G., Pradera, A., Calvo, T. (2007). Aggregation Functions: A Guide for Practitioners. Springer, Heidelberg, Berlin, New York.

Beliakov, G., Bustince, H., Goswami, D.P., Mukherjee, U.K., Pal, N.R. (2011). On averaging operators for Atanassov's intuitionistic fuzzy sets. Information Sciences, 181, 1116-1124.

Bonferroni, C. (1950). Sulle medie multiple di potenze. Bolletino Matematica Italiana, 5, 267-270. 
Calvo, T., Mesiar, R. (2003). Weighted triangular norms-based aggregation operators. Fuzzy Sets and Systems, 137, 3-10.

Chen, T.Y. (2011). Bivariate models of optimism and pessimism in multi-criteria decision-making based on intuitionistic fuzzy sets. Information Sciences, 181, 2139-2165.

Chen, S.M., Tan, J.M. (1994). Handling multicriteria fuzzy decision-making problems based on vague set theory. Fuzzy Sets and Systems, 67, 163-172.

Del Moral, M.J., Chiclana, F., Tapia, J.M., Herrera-Viedma, E. (2018). A comparative study on consensus measures in group decision making. International Journal of Intelligent Systems, 33, 1624-1638.

Deng, J.L. (1989). Introduction to grey system theory. Journal of Grey Systems, 1, 1-24.

Deschrijver, G., Kerre, E.E. (2003). On the relationship between some extensions of fuzzy set theory. Fuzzy Sets and Systems, 133, 227-235.

Deschrijver, G., Kerre, E.E. (2007). On the position of intuitionistic fuzzy set theory in the framework of theories modelling imprecision. Information Sciences, 177, 1860-1866.

Deschrijver, G., Kerre, E.E. (2008). Aggregation operators in interval-valued fuzzy and Atanassov's intuitionistic fuzzy set sheory. In: Bustince, H., Herrera, F., Montero, J. (Eds.), Fuzzy Sets and Their Extensions: Representation, Aggregation and Models. Springer, Heidelberg, New York, pp. 183-203.

Dong, Y., Zhao, S., Zhang, H., Chiclana, F., Herrera-Viedma, E. (2018). A self-management mechanism for non-cooperative behaviors in large-scale group consensus reaching processes. IEEE Transactions on Fuzzy Systems, 26, 3276-3288.

Dubois, D., Gottwald, S., Hajek, P., Kacprzyk, J., Prade, H. (2005). Terminological difficulties in fuzzy set theory - the case of intuitionistic fuzzy sets. Fuzzy Sets and Systems, 156, 485-491.

Gau, W.L., Buehrer, D.J. (1993). Vague sets. IEEE Transactions on Systems Man and Cybernetics, 23, $610-614$.

Hardy, G.H., Littlewood, Pólya G, J.E. (1934). Inequalities. Cambridge University Press, Cambridge.

Hong, D.H., Choi, C.H. (2000). Multicriteria fuzzy decision-making problems based on vague set theory. Fuzzy Sets and Systems, 114, 103-113.

Klement, E.P., Mesiar, R., Pap, E. (2000). Triangular Norms. Kluwer Academic Publishers, Boston, London, Dordrecht.

Li, D.F. (2010). Multiattribute decision making method based on generalized OWA operators with intuitionistic fuzzy sets. Expert Systems with Applications, 37, 8673-8678.

Liao, H., Xu, Z.S. (2014). Intuitionistic fuzzy hybrid weighted aggregation operators. International Journal of Intelligent Systems, 29, 971-993.

Liao, H., Xu, Z.S. (2015). Consistency of the fused intuitionistic fuzzy preference relation in group intuitionistic fuzzy analytic hierarchy process. Applied Soft Computing, 35, 812-826.

Ma, Z.M., Xu, Z.S. (2016). Symmetric Pythagorean fuzzy weighted geometric/averaging operators and their application in multicriteria decision-making problems. International Journal of Intelligent Systems, 31, 11981219.

Ma, Z.M., Xu, Z.S. (2018). Hyperbolic scales involving appetites-based intuitionistic multiplicative preference relations for group decision making. Information Sciences, 451-452, 310-325.

Sambuc, R. (1975). Fonctions $\phi$-floues. Application l'aide au diagnostic en pathologie thyroidienne. Ph.D. Thesis, Univ. Marseille, France.

Tan, C., Jiang, Z., Chen, X. (2013). Some issues on quasi-arithmetic intuitionistic fuzzy OWA operators. Applied Mathematics \& Information Sciences, 7, 955-961.

Urena, R., Kou, G., Dong, Y., Chiclana, F., Herrera-Viedma, E. (2019). A review on trust propagation and opinion dynamics in social networks and group decision making frameworks. Information Sciences, 478, 461-475.

Wang, W., Liu, X. (2011). Intuitionistic fuzzy geometric aggregation operators based on Einstein operations. International Journal of Intelligent Systems, 26, 1049-1075.

Wang, W., Liu, X. (2012). Intuitionistic fuzzy information aggregation using Einstein operations. IEEE Transactions on Fuzzy Systems, 20, 923-938.

Xu, Z.S. (2007). Intuitionistic fuzzy aggregation operators. IEEE Transactions on Fuzzy Systems, 15, 1179-1187.

$\mathrm{Xu}, \mathrm{Z} . S$., Yager, R.R. (2006). Some geometric aggregation operators based on intuitionistic fuzzy sets. International Journal of Intelligent Systems, 35, 417-433.

Xu, Z.S., Yager, R.R. (2011). Intuitionistic fuzzy Bonferroni means. IEEE Transactions on Systems, Man, and Cybernetics-Part B: Cybernetics, 41, 568-578.

Xu, C.Y., Ma, Z.M. (2019). Symmetric intuitionistic multiplicative aggregation operator for group decision making in intuitionistic multiplicative environments. Journal of Intelligent \& Fuzzy Systems, 36, 5909-5918. 
Xia, M., Xu, Z.S. (2012). Entropy/cross entropy-based group decision making under intuitionistic fuzzy environment. Information Fusion, 13, 31-47.

Xia, M., Xu, Z.S., Zhu, B. (2012). Some issues on intuitionistic fuzzy aggregation operators based on Archimedean $t$-conorm and $t$-norm. Knowledge-Based Systems, 31, 78-88.

Yager, R.R. (1988). On ordered weighted averaging aggregation operators in multi-criteria decision making. IEEE Transactions on Systems Man and Cybernetics, 18, 183-190.

Yager, R.R. (2004a). Weighted triangular norms using generating functions. International Journal of Intelligent Systems, 19, 217-231.

Yager, R.R. (2004b). Generalized OWA aggregation operators. Fuzzy Optimization and Decision Making, 3, 93-107.

Yager, R.R. (2009). On generalized Bonferroni mean operators for multi-criteria aggregation. International Journal of Approximate Reasoning, 50, 1279-1286.

Yager, R.R., Filev, D.P. (1999). Induced ordered weighted averaging operators. IEEE Transactions on Systems Man and Cybernetics, 29, 141-150.

Yang, W., Chen, Z.P. (2012). The quasi-arithmetic intuitionistic fuzzy OWA operators. Knowledge-Based Systems, 27, 219-233.

Yang, W., Jhang, S.T., Fu, Z.W., Ma, Z.M. (2019). Aggregating intuitionistic fuzzy preference relations with symmetrical intuitionistic fuzzy Bonferroni mean operators in group decision making. Information Sciences.

Zadeh, L.A. (1965). Fuzzy sets. Information Control, 8, 338-353.

Zadeh, L.A. (1975). The concept of a linguistic variable and its application to approximate reasoning. Information Sciences, 8, 199-249.

Zhao, H., Xu, Z.S., Ni, M.F., Liu, S.S. (2010). Generalized aggregation operators for intuitionistic fuzzy sets. International Journal of Intelligent Systems, 25, 1-30. 
Z.M. Ma is an associate professor at School of Mathematics and Statistics, Linyi University. He has received his MS degree in systems theory and $\mathrm{PhD}$ in computational mathematics from the School of Mathematics and Statistics, Wuhan University. His current research is focused on decision-making theory and intelligent decision support systems.

W. Yang is a lecturer at School of Mathematics and Statistics, Linyi University. She has received her MS degree in applied mathematics from the School of Scineces, University of Science and Technology, Beijing. Her current research is focused on decision-making theory. 